\title{
REGIONAL INFLUENCES ON ALGAL BIODIVERSITY IN TWO POLLUTED RIVERS OF EURASIA (RUDNAYA RIVER, RUSSIA, AND QISHON RIVER, ISRAEL) BY BIOINDICATION AND CANONICAL CORRESPONDENCE ANALYSIS
}

\author{
S. BARINOVA ${ }^{1} *-$ L. MEDVEDEVA $^{2}-$ E.NEVO $^{1}$ \\ ${ }^{1}$ The Algology Laboratory in the Institute of Evolution, University of Haifa \\ Mount Carmel, Haifa 31905 Israel \\ ${ }^{2}$ Institute of Biology and Soil Science, Far-East Branch of Russian Academy of Science, \\ Stoletiya Str., 159, Vladivostok 690022 Russia \\ (phone: +972-4-8249697; fax: +972-4-8246554) \\ *Corresponding author \\ e-mail: barinova@research.haifa.ac.il \\ (Received 29 ${ }^{\text {th }}$ August 2007; accepted $1^{\text {st }}$ March 2008)
}

\begin{abstract}
In our study we investigated two polluted rivers of Eurasia from silicate and carbonate regions. We revealed an algal diversity consisting of 184 algal species and cyanoprokaryotes in the Rudnaya River, and 175 in the Qishon River. The distributions of species over the 7 higher taxa were very similar for both rivers with diatom prevealing. Bioindicational analysis in respect salinity, acidification, oxygenation, and organic pollution show that the water is cleaner and the diversity is higher in the Rudnaya River than in the Qishon River. The indices of saprobity S ranged similar. The impact of pollution on Rudnaya River increases downstream. In tha case of Qishon River, the impact of pollution decreases downstream.As a result of CCA, we revealed biosensors group sensitive to borates and fluorides in the case of Rudnaya River and these were: Ankistrodesmus acicularis, A. angustus, Scenedesmus acuminatus, Lyngbya kuetzingii, Neidium ampliatum, and Sellaphora rectangularis. In the case of Qishon River, Audouinella pygmaea, Characium ornithocephalum, and Chamaesiphon amethystinus were found as biosensor species. We found that algal biodiversity is more sensitive to technogenic pollution in the silicate province being more tolerant to the same organic pollutants in the carbonate province. Therefore, the combination of bioindicational methods and statistics are effective for determination of the main factors influencing algal diversity, indicators or biosensing species for the most important environmental variables.
\end{abstract}

Keywords: CCA, algal biodiversity, ecology, Russia, Israel.

\section{Introduction}

According to our research, algal diversity is significantly influenced by changes in the environmental parameters. We can use this contiguity in bioindication methodology. However, the major parameters used in bioindication are individual for each species, while the reaction of the entire community is left unseen.

To change this situation, in this research we reveal the reaction of the entire algal community present in two rivers in Eurasia, the Rudnaya River and the Qishon River (Figure 1) as a result of the changes in water parameters under anthropogenic pollution and regional climatic influence.

The main characteristic of the Israeli climate is a short rainy season,which lasts from December to March. The cliamte of the Far-East is mainly characterized by a summerhumidity season which lasts from April to October. 
Therefore, we have assumed that water quality during the rainy season can represent the sum of all climatic influences and anthropogenic impacts in both regions. The bioindicational analysis is able to define the major influencing factors. Oridnation as a statistical method can also be used if bioindicational analysis fails to give the desired results. This method can be used to show, for example, the unique light and temperature responses of cyanobacterial species from two differens habitats on Antarctic and Arizona (Banerjee, Sharma 2004).

\section{Study sites description}

\section{Rudnaya River}

The Russian Far East is basically a basaltic volcanic area (Meybeck \& Helmer 1989). The Rudnaya River starting at the eastern slope of Sikhote-Alin Range and it empties into the Rudnaya bay of the Sea of japan. The river is $73 \mathrm{~km}$ long, its basin occupies 1140 square kilometre. The water flow varies over the year: 96 percent during the warm season, from April to November, and only 4 percent during the cold period, from December to March (Berezneva 2006).

The Rudnaya River Basin is ecologically the most polluted area in the Primorye Region. Ore mines, enrichment factories and other industries contribute to the pollution in the river valley.

Both surface and underground waters are heavily polluted and lost their utility as a water source over the river flow from Krasnorechensk to Rudnaya Pristan'. The Rudnaya River had lost its significance as fishing water (Kuznetsova 2005).

About 20,000 tons of various technogenic substances are discharged to the river per anuum. The concentration of $\mathrm{Mn}, \mathrm{Zn}, \mathrm{Cd}$ and $\mathrm{Pb}$ in the polluting substances is more than ten times higher than their natural level. The residents suffer from serious lead poisoning caused by the old smelters and the insecure transporation of the lead concentrate from the local mining site.

The sources of water pollution can be grouped according to their origin and their impact. However, an objective information on the pollution sources is lacking. The tentative assessments given below are lesser than real. The discharges, in t/year are the following: $\mathrm{Cu}-0.13$; Zn-4.66; Fe-1.12; Pb-0.49 (Kuznetsova 2005).

According to the most recent study, lead concentrations in the residential gardens $(476-4310 \mathrm{mg} / \mathrm{kg}$ ) and in roadside soils $(2020-22900 \mathrm{mg} / \mathrm{kg})$ exceed USEPA regulations by orders of magnitude. These data suggest that drinking water, dust in the air, and garden plants also likely contain dangerous levels of lead. Water discharged from the smelter $2900 \mathrm{~m}^{3}$ as a daily average with concentrations up to $100 \mathrm{~kg}$ of lead and $20 \mathrm{~kg}$ of arsenic (Von Braun et al. 2002; Kachur et al. 2003; Sharov 2005; Shilov 1997).

\section{Qishon River}

The Qishon River is the second largest river among the coastal rivers of Israel, and also regarded as the most polluted river system in Israel (Herut et al., 2000; Herut \& Kress 1997). In general, $\mathrm{pH}$ is relatively constant fluctuating between 9.1 and 7.5 down the river channel corresponding to the regional norm for carbonate provinces (Meybeck $\&$ Helmer 1989). The river drains an area of approximately $1100 \mathrm{~km}^{2}$ and flows through agricultural, domestic, and industrial districts before joining the Mediterranean Sea near 
the city of Haifa. The Qishon's water is contaminated by agricultural runoff, various types of industrial effluents, and domestic sewage (Herut \& Kress 1997); by heavy metals and a mixture of organic materials (Richter et al. 2003; Herut et al. 1994; Krumgalz 1993) including polycyclic aromatic hydrocarbons (PAHs), alkylated benzenes, halogenated alkanes, and chlorinated aromatic organic compounds; and some radionucleotides. As a result, the lower part of the Qishon River has been denuded for years of its multicellular life forms (Richter et al. 2003). Moreover, tissue analyses of fishes, crustaceans, and mollusks from the shallow waters of Haifa Bay at the mouth of Qishon River revealed high levels of heavy metals and deviations in oxidizing enzyme activities (Kress et al. 1999; Herut et al. 1999). Herut et al. (2003) reported medium degree of pollution in the Qishon estuary by heavy metals (mercury, copper, zinc, cadmium, nickel) and high degree of pollution upstream by international environmental criteria. Not surprisingly, the Qishon River has been identified as the main source of mercury, cadmium and other heavy metals pollution in the Haifa ports. High concentrations of Tributyltin (TBT) $(>100 \mu \mathrm{g} / \mathrm{Kg})$, probably originating from antifouling paints, were found in the sediments at both, the Haifa port, and Qishon river. Probably, as the consequences of the TBT pollution the entire female population of the snail Murex forskoehli collected in the vicinity of Haifa Port ( 800 specimens) exhibited male sexual characteristics. The sediments at the Ports of Haifa, and Qishon river are also significantly contaminated by PCB's and by polycyclic aromatic pollutants (PAH's) not to speak about high levels (recorded from 1990 to 2002) of dissolved inorganic nutrients (nitrogen and phosphorous), representing a high degree of pollution by international environmental criteria (Herut et al. 2003). The Qishon River has also elicited major public concern for its potential long-term cancer risks to fisherman working in its vicinity and to navy soldiers who used to dive in the river (Richter et al. 2003).

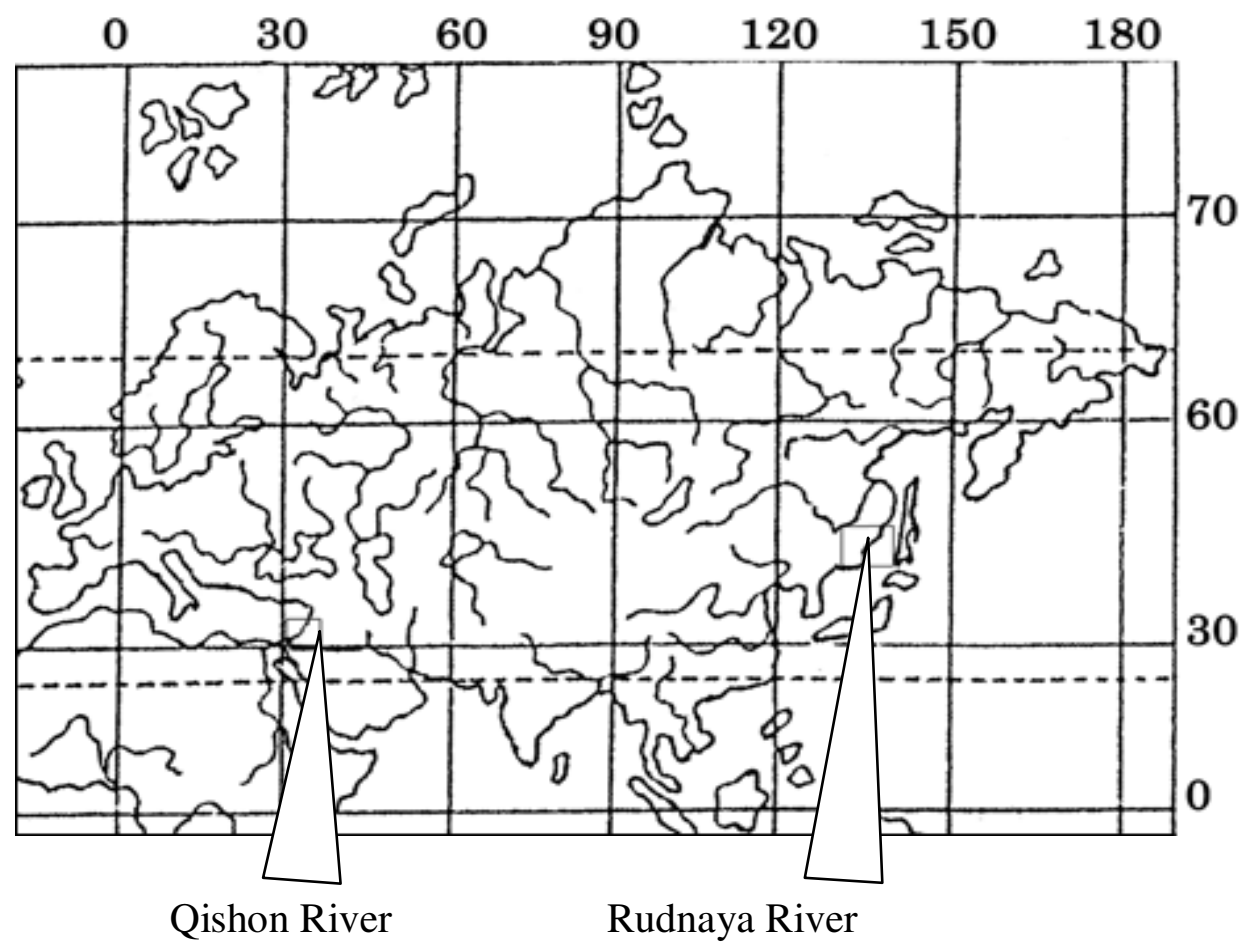

Figure 1. Map of the study site. 
a)

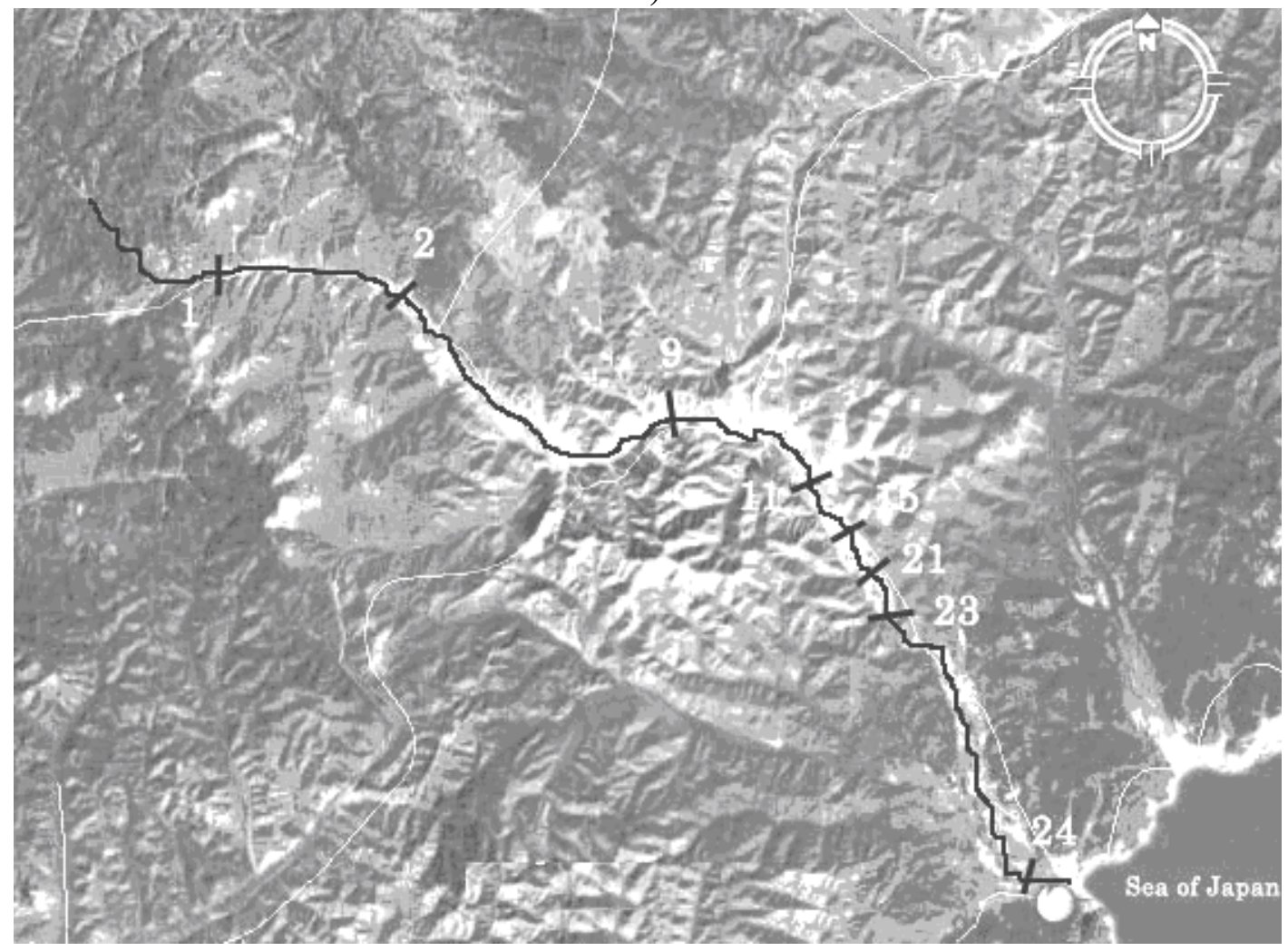

b)

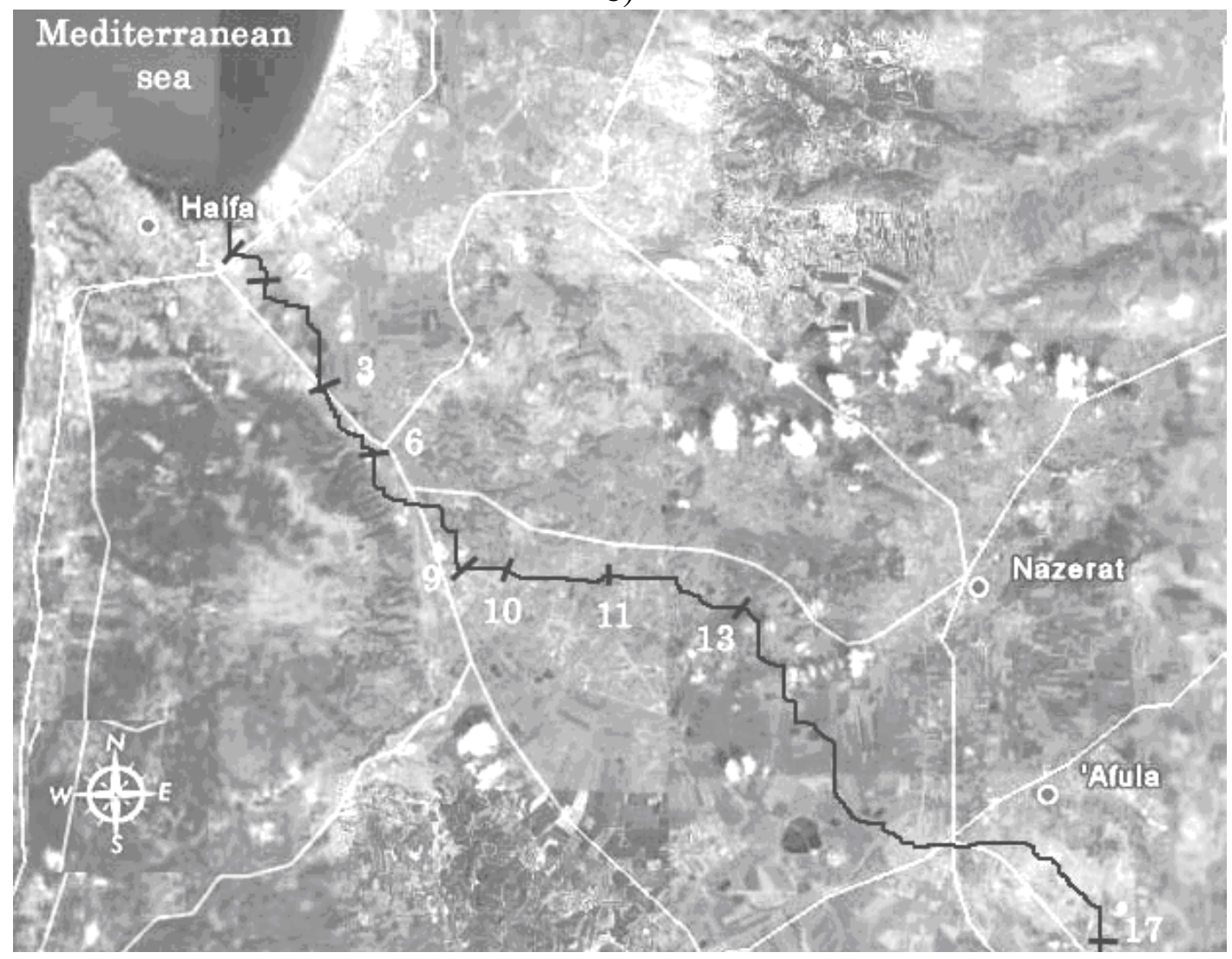

Figure 2. Sampling stations over the Rudnaya River (a) and Qishon River (b). 


\section{Materials and methods}

\section{Materials}

For our study we have collected 649 samples of planktonic and periphytonic algae from 24 stations over the Rudnaya River in 1978-1990 (Figure 2a), as well as 117 algological samples from 19 stations over the Qishon River (Figure 2b) that were collected during the winter and summer seasons, from February 2002 to November 2005.

The samples were obtained by scooping up for phytoplankton and by scratching for periphyton and were fixed in 3\% formaldehyde. Algae were studied with a dissecting Swift and Amplival microscopes under magnifications of 740-1850 and were photographed with the digital camera Inspector 1 . The diatoms were prepared with the peroxide technique (Swift 1967) modified for glass slides (Barinova 1997). The diatoms were studied both under light microscope and scanning electron microscope JEOL JSM 35C. The taxonomy of this study mainly follows the systems adopted in the "Süswasserflora von Mitteleuropa".

Table 1. shows the chemical data for Rudnaya River come from Elpatievsky et al. (1976).

In parallel with sampling for algae in Qishon River, we measured conductivity, mineralization and $\mathrm{pH}$ with HANNA HI 9813. In addition to our sampling we have used data of chemical analysis regularly performed by "Mekorot Water Co." shown in Table 2.

Table 1. Chemical variables over the Rudnaya River stations.

\begin{tabular}{c|c|c|c|c|c|c|c|c}
\hline Station & $\mathbf{1}$ & $\mathbf{2}$ & $\mathbf{9}$ & $\mathbf{1 1}$ & $\mathbf{1 5}$ & $\mathbf{2 1}$ & $\mathbf{2 3}$ & $\mathbf{2 4}$ \\
\hline $\mathrm{pH}$ & 7.54 & 7.54 & 7.56 & 7.56 & 7.72 & 8.3 & 8.3 & 7.61 \\
$\mathrm{HCO}_{3}^{-}, \mathrm{mg} / \mathrm{l}$ & 39.0 & 39.0 & 40.0 & 40.0 & 52.0 & 63.0 & 63.0 & 40.0 \\
$\mathrm{Cl}^{-}, \mathrm{mg} / \mathrm{l}$ & 4.9 & 4.9 & 7.2 & 7.2 & 6.4 & 12.7 & 12.7 & 8.6 \\
$\mathrm{SO}_{4}{ }^{3-}, \mathrm{mg} / \mathrm{l}$ & 11.8 & 11.8 & 27.5 & 27.5 & 24.0 & 116.0 & 116.0 & 35.5 \\
$\mathrm{Ca}^{2+}, \mathrm{mg} / \mathrm{l}$ & 9.0 & 9.0 & 17.0 & 17.0 & 16.3 & 63.0 & 63.0 & 11.0 \\
$\mathrm{Mg}^{2+}, \mathrm{mg} / \mathrm{l}$ & 2.1 & 2.1 & 4.5 & 4.5 & 4.0 & 3.8 & 3.8 & 2.5 \\
$\mathrm{~K}^{+}, \mathrm{mg} / \mathrm{l}$ & 0.5 & 0.5 & 1.2 & 1.2 & 1.1 & 1.8 & 1.8 & 1.4 \\
$\mathrm{Na}^{+}, \mathrm{mg} / \mathrm{l}$ & 3.5 & 3.5 & 5.3 & 5.3 & 5.2 & 13.2 & 13.2 & 6.7 \\
$\mathrm{SiO}_{2}, \mathrm{mg} / \mathrm{l}$ & 7.2 & 7.2 & 6.8 & 6.8 & 5.8 & 6.3 & 6.3 & 5.2 \\
$\mathrm{~B}_{2} \mathrm{O}_{3}, \mathrm{mg} / \mathrm{l}$ & 0 & 0 & 0 & 0 & 0 & 9.4 & 9.4 & 1.4 \\
$\mathrm{~F}, \mathrm{mg} / \mathrm{l}$ & 0.06 & 0.06 & 0.1 & 0.1 & 0.1 & 0.42 & 0.42 & 0.17 \\
$\mathrm{TDS}, \mathrm{mg} / \mathrm{l}$ & 71.2 & 71.2 & 110.6 & 110.6 & 100.4 & 272.2 & 272.2 & 118 \\
$\mathrm{Cu}, \mathrm{mkg} / \mathrm{l}$ & 2.2 & 2.2 & 6.0 & 6.0 & 2.5 & 8.3 & 8.3 & 9.7 \\
$\mathrm{Zn}, \mathrm{mkg} / \mathrm{l}$ & 16.5 & 16.5 & 180.3 & 180.3 & 47.8 & 78.7 & 78.7 & 100.0 \\
$\mathrm{~Pb}, \mathrm{mkg} / 1$ & 5.7 & 5.7 & 17.0 & 17.0 & 10.4 & 30.8 & 30.8 & 40.5 \\
$\mathrm{Sn}, \mathrm{mkg} / \mathrm{l}$ & 4.5 & 4.5 & 5.9 & 5.9 & 3.0 & 8.5 & 8.5 & 19.7 \\
$\mathrm{Ag}, \mathrm{mkg} / \mathrm{l}$ & 0.8 & 0.8 & 0.2 & 0.2 & 0.5 & 0.6 & 0.6 & 0.8 \\
$\mathrm{As}, \mathrm{mkg} / \mathrm{l}$ & 0 & 0 & 1.8 & 1.8 & 1.5 & 4.4 & 4.4 & 5.5 \\
$\mathrm{Index} \mathrm{S}$ & 1.32 & 1.76 & 2.22 & 2.18 & 2.05 & 2.26 & 2.13 & 2.18 \\
$\mathrm{No}$ of Species & 35 & 13 & 26 & 16 & 15 & 24 & 17 & 22 \\
\hline
\end{tabular}


Table 2. Chemical variables over the Qishon River stations.

\begin{tabular}{|c|c|c|c|c|c|c|c|c|c|}
\hline Station & 17 & 13 & 11 & 10 & 9 & 6 & 3 & 2 & 1 \\
\hline $\mathrm{T}$ & 22.4 & 22.8 & 24.7 & 25.8 & 25.8 & 24.7 & 24.8 & 29.4 & 30 \\
\hline $\mathrm{pH}$ & 7.89 & 7.83 & 8.07 & 7.91 & 7.85 & 8.05 & 7.97 & 8.1 & 8.58 \\
\hline $\mathrm{E} \mathrm{ms} / \mathrm{cm}$ & 3.56 & 6.05 & 5.5 & 5.11 & 4.87 & 5.04 & 4.93 & 9.42 & 9.97 \\
\hline TDS, mg/l & 4000 & 6000 & 5000 & 5000 & 4500 & 5000 & 4500 & 8000 & 8000 \\
\hline TSS 105 & 322 & 172 & 133 & 68 & - & 114 & 200 & 36 & 74 \\
\hline $\mathrm{O}_{2,} \mathrm{mg} / \mathrm{l}$ & 8.8 & 8.2 & 9.4 & 11.1 & 8.5 & 9.1 & 6.8 & 20.1 & 29.6 \\
\hline Saturated & 98 & 92 & 105 & 136 & 104 & 110 & 90 & 235 & 370 \\
\hline Sulfides, mg/l & 0 & 0.14 & 1.8 & 0.42 & 0 & 1.2 & 0 & 0.04 & 0.51 \\
\hline $\mathrm{PO}_{4}^{+}, \mathrm{mg} / \mathrm{l}$ & 0 & 0 & 0.3 & 0 & 0 & 0.4 & 0 & 0 & 1.2 \\
\hline Total P, mg/l & 0.5 & 0.6 & 0.5 & 0.9 & - & 0.7 & 0.6 & 1.9 & 2.3 \\
\hline $\mathrm{NO}_{3}, \mathrm{mg} / \mathrm{l}$ & 27 & 13.4 & 11.3 & 6 & 8.1 & 6.2 & 7.2 & 11.1 & 6 \\
\hline $\mathrm{NO}_{2}^{-}, \mathrm{mg} / \mathrm{l}$ & 0.1 & 0.087 & 0.006 & 0.35 & - & 0.33 & 0.059 & 0.33 & 0.33 \\
\hline $\mathrm{NH}_{4}^{+}, \mathrm{mg} / \mathrm{l}$ & 0.05 & 0.05 & 0.05 & 0.05 & - & 0.05 & 0.05 & 1.3 & 0.6 \\
\hline $\mathrm{N}$ org., $\mathrm{mg} / \mathrm{l}$ & 2.5 & 1.5 & 1.8 & 2.9 & - & 1.7 & 2.3 & 4.7 & 5.7 \\
\hline Total N & 29.6 & 15 & 13.2 & 9.3 & - & 8.2 & 9.6 & 16.1 & 12.03 \\
\hline BOD, mg/l & 9 & 6.3 & 6 & 11.1 & - & 10.2 & 8 & 12.3 & 15.3 \\
\hline Min. oil, mg/l & 0.5 & 0.5 & 0.5 & 0.5 & 0.5 & 0.5 & - & 0.5 & 0.5 \\
\hline Total oil, mg/l & 0.5 & 0.5 & 0.5 & 0.5 & 0.5 & 0.5 & - & 0.5 & 0.7 \\
\hline E. Coli t/l & 3300 & 9000 & 3500 & 14000 & - & 24000 & 12000 & 12000 & 500 \\
\hline $\mathrm{Chl} a, \mathrm{mg} / \mathrm{l}$ & 0.005 & 0.021 & 0.013 & 0.024 & - & 0.021 & 0.005 & 0.093 & 0.021 \\
\hline $\mathrm{Cl}^{-}, \mathrm{mg} / \mathrm{l}$ & - & - & 1306 & - & - & 773 & - & 10263 & 11663 \\
\hline $\mathrm{COD}, \mathrm{mg} / \mathrm{l}$ & - & - & 32 & - & - & 58 & - & 49 & 58 \\
\hline $\mathrm{TOC}, \mathrm{mg} / \mathrm{l}$ & - & - & 8.8 & - & - & 14.2 & - & 13.6 & 15 \\
\hline $\begin{array}{l}\text { Detergents, } \\
\mathrm{mg} / \mathrm{l}\end{array}$ & - & - & 0.2 & - & - & 0.24 & - & 0.17 & 0.26 \\
\hline Benzene, $\mathrm{mg} / \mathrm{l}$ & - & - & 0.05 & - & - & 0.05 & - & 0.05 & - \\
\hline Toluol, mg/l & - & - & 0.05 & - & - & 0.05 & - & 0.05 & - \\
\hline Xylen, mg/l & - & - & 0.05 & - & - & 0.05 & - & 0.05 & - \\
\hline Phenol, mg/l & - & - & 0.002 & - & - & 0.002 & - & 0.002 & - \\
\hline microtox & - & - & 22 & - & - & 13 & - & 0 & - \\
\hline $\begin{array}{c}\text { Chlorine, } \\
\mathrm{mg} / \mathrm{l}\end{array}$ & - & - & 0.06 & - & - & 0.06 & - & - & - \\
\hline Index S & 2.51 & 2.49 & 2.48 & 2.03 & 2.31 & 2.2 & 2.53 & 2.33 & 2.31 \\
\hline No of Species & 20 & 28 & 26 & 36 & 32 & 28 & 18 & 9 & 17 \\
\hline
\end{tabular}

Density scores were calculated using a 5-score scale (Whitton \& al. 1991) and 6score scale (Korde 1956). Saprobity indices were obtained for each algal community (Pantle \& Buck 1955; Sladeček 1986) and then used for integral assessment of the species habitats. Ecological and horological characteristics of the species are summed up in our database (Barinova \& al. 2006).

Our ecological analysis revealed freshwater algae ecological groups in respect to $\mathrm{pH}$, salinity, and saprobity, as well as temperature, streaming, and oxygenation. Each group was separately assessed in respect to its significance for bioindication. Species that respond predictably to these variables can be used as bioindicators reflecting the response of aquatic ecosystems to eutrophication, $\mathrm{pH}$ levels (acidification), salinity, and organic pollutants.

Diagrams of species distribution were constructed for each group of ecological indicators. The algal groups were ordinated according to their increasing tolerances for a given environmental variable. A polynomial/linear trend line was defined (as a 
statistic function in the Microsoft EXCEL program) for distribution of algal groups in response to individual variables, showing the general tendency of the diversity changes in respect to fluctuations of a given variable.

The distribution in the number of species between the groups in the different indicator systems shows the total range of environmental conditions in the river, on one hand, and the prevailing conditions, on the other. The summit of the trend line corresponds to the optimal conditions in respect to the given variable.

Out of the many possible the ordination methods available we have chosen to use Canonical Correspondence Analysis (CCA). We were able to obtain quantitative information on the relationship between species and environmental variables using CCA with the CANOCO for Windows 4.5 package.

Estimation of the explanatory power for each environmental variable was performed using the variable as the sole constraining variable. Statistical significance for each variable was assessed using the Monte Carlo unrestricted permutation test involving 999 permutations (ter Braak 1987; 1990).

The CCA biplot represents the ordination of species in relation to the combination of the different environmental variables. Environmental variables are represented by arrows, the maximal value for each variable is located at the arrow head. Therefore, species marked near arrow head are bioindicators in respect this variable. Species marked near opposite end of arrow are biosensors, which is sensitive even to presence of given variable.

\section{Results and discussion}

\section{Rudnaya River}

Data on water hydrochemistry of the Rudnaya River are cited in table I (Elpatievsky et al. 1976). Unfortunately, the existing data pertain to metal concentrations only except the data on stations 1 and 2, which show normal distribution of chemical elements in the natural unpolluted water sources in the Russian Far East. Insignificant $\mathrm{pH}$ fluctuations suggest that the river waters are fresh over the most of the watercourse. Even at the mouth station $24 \mathrm{pH}$ does not exceed 7.6. An increase of $\mathrm{pH}$ up to 8.3 was measured at stations 21 and 23 only. These stations are situated down current of the polluting tributaries of the Rudnaya River.

The analysis of table 1 shows that concentrations of $\mathrm{HCO}_{3}{ }^{-}, \mathrm{Cl}^{-}, \mathrm{Ca}^{2+}, \mathrm{Na}^{+}, \mathrm{TDS}, \mathrm{Cu}$, $\mathrm{Pb}, \mathrm{Sn}$ in the Rudnaya River, in general and at the station 21 and 23 in particular are higher than the background level. The concentration of borates, sulfates, and fluorides increase up to ten times at these stations. Notable also is a significant increase of $\mathrm{Zn}$ at the stations 9 and 11 .

As it was expected, the saprobity index is the lowest at the clear water station 1 $(\mathrm{S}=1.32)$ up current of the major pollution sources. Similar values of saprobity indices are obtained for the majority of water sources in the Russian Far East (Barinova \& Medvedeva 1987). The influences of polluting discharges are reflected in the increase in the saprobity index up to 2.18-2.26. The maximal biological diversity of algal communities was observed at the clear-water station 1, appreciably decreasing down the current. 


\section{Qishon River}

The physico-chemical environmental variables and their dynamics over the Qishon River stations are indicated in Table 2. The $\mathrm{pH}$ and conductivity show that Qishon River is fresh water in the upper and middle reaches, is influenced by sea tides in the lower reaches (chloride fluctuated from $773 \mathrm{mg}$ per liter to $11663 \mathrm{mg}$ per liter (Qishon River Authority http://www.kishon.org.il/) and remains alkaline all year-round. The concentration of $\mathrm{N}^{-\mathrm{NO}_{3}}$ is $0.6-10.5 \mathrm{mg}$ per liter. The concentration of P-total is $0.2-7.93$ $\mathrm{mg}$ per liter. In winter, conductivity tends to increase from station 10 to the mouth, while $\mathrm{pH}$ tends to decrease in the same direction. Connection between conductivity and salinity levels as well as other chemical variables and Index of saprobity $\mathrm{S}$ indicated in the Table 2. In general, $\mathrm{pH}$ in Qishon River is relatively constant fluctuating between 9.1 and 7.5 down the river channel corresponding to the regional norm for carbonate provinces (Meybeck \& Helmer 1989).

In samples from Qishon River we found 175 species of algae from 7 divisions (Barinova et al. 2004; 2006). In samples from Rudnaya River we found 184 species of algae from 7 divisions (Medvedeva et al. 1986a,b; Medvedeva \& Barinova 1992; Barinova \& Medvedeva 1989, 1992a,b). As we can see on the Table 3, the species diversity in each river is significantly different, that reflects their regional ecological preferences. The comparison of found diversity (Table 3) is on the Figure 3.

Table 3. Abundance and ecological preference of algal species revealed in the Rudnaya River and Qishon River.

\begin{tabular}{|c|c|c|c|c|c|c|c|c|c|c|c|}
\hline No & Taxon & Code & Rud & Qish & Hab & $\mathbf{T}$ & Oxy & D & $\mathbf{S}$ & Hal & pH \\
\hline & Cyanoprokaryota & & & & & & & & & & \\
\hline 1 & Anabaena spiroides Kleb. & ANSPIH & 0 & $1-3$ & $\mathrm{P}$ & - & $\begin{array}{l}\text { st- } \\
\text { str }\end{array}$ & - & $\mathrm{o}$ & $\mathrm{i}$ & - \\
\hline 2 & $\begin{array}{l}\text { Anabaena variabilis } \mathrm{f} . \\
\text { rotundospora Hollerb. }\end{array}$ & ANAVRQ & 0 & $1-6$ & B & - & - & - & - & - & - \\
\hline 3 & Anabaena sp. & AN001Y & 0 & $1-3$ & - & - & - & - & - & - & - \\
\hline 4 & $\begin{array}{l}\text { Aphanocapsa elachista } \\
\text { W. et G.S. West }\end{array}$ & MICPUR & 1 & 0 & - & - & - & - & - & - & - \\
\hline 5 & $\begin{array}{l}\text { Aphanothece elabens } \\
\text { (Bréb.) Elenk. }\end{array}$ & APHACQ & 0 & 2 & P-B & - & - & - & - & - & - \\
\hline 6 & $\begin{array}{l}\text { Aphanothece } \\
\text { microscopica Näg. }\end{array}$ & APHAMR & 2 & 0 & B & temp & - & - & $\mathrm{o}$ & $\mathrm{hb}$ & \\
\hline 7 & $\begin{array}{l}\text { Aphanothece stagnina } \\
\text { (Spreng.) A. Braun in } \\
\text { Rabenh. }\end{array}$ & ATS01Y & 0 & 1 & P-B & - & - & - & b-a & $\mathrm{hl}$ & ind \\
\hline 8 & $\begin{array}{l}\text { Arthrospira fusiformis } \\
\text { (Woronich.) Komárek et } \\
\text { Lund }\end{array}$ & ARTHSQ & 0 & 1 & $\mathrm{P}$ & - & - & - & - & $\mathrm{mh}$ & - \\
\hline 9 & $\begin{array}{l}\text { Calothrix fusca f. parva } \\
\text { (Erceg.) V. Poljansk. }\end{array}$ & CAFUSR & 6 & 0 & - & - & - & - & - & - & - \\
\hline 10 & $\begin{array}{l}\text { Chamaesiphon } \\
\text { amethystinus (Rostaf.) } \\
\text { Lemm. }\end{array}$ & CHAMAQ & 0 & $1-5$ & Ep & - & str & - & - & - & - \\
\hline 11 & $\begin{array}{l}\text { Chroococcus minor } \\
\text { (Kütz.) Näg. }\end{array}$ & GLOCAR & 1 & 0 & B & - & - & - & $o-b$ & - & - \\
\hline 12 & $\begin{array}{l}\text { Crinalium endophyticum } \\
\text { Crow }\end{array}$ & CRINAQ & 0 & 1 & Ep & - & - & - & - & - & - \\
\hline 13 & $\begin{array}{l}\text { Hydrococcus rivularis } \\
\text { (Kütz.) Menegh. }\end{array}$ & HYDRCQ & 0 & 4 & B & - & - & - & $\mathrm{x}$ & - & - \\
\hline
\end{tabular}




\begin{tabular}{|c|c|c|c|c|c|c|c|c|c|c|c|}
\hline No & Taxon & Code & Rud & Qish & Hab & $\mathbf{T}$ & Oxy & D & $\mathbf{S}$ & Hal & pH \\
\hline 14 & $\begin{array}{l}\text { Limnothrix } \\
\text { amphigranulata (Van } \\
\text { Goor) Meffert }\end{array}$ & LIA01Y & 0 & 6 & B & - & st & - & - & $\mathrm{mh}$ & - \\
\hline 15 & $\begin{array}{l}\text { Lyngbya kuetzingii } \\
\text { Schmidle }\end{array}$ & LYNKUL & 3 & 0 & B & - & $\begin{array}{l}\text { st- } \\
\text { str }\end{array}$ & - & $o-b$ & - & - \\
\hline 16 & $\begin{array}{l}\text { Lyngbya limnetica } \\
\text { Lemm. }\end{array}$ & LYL01Y & 0 & 2 & P-B & - & $\begin{array}{l}\text { st- } \\
\text { str }\end{array}$ & - & $o-b$ & hl & - \\
\hline 17 & $\begin{array}{l}\text { Lyngbya pusilla } \\
\text { (Rabenh.) Hansg. }\end{array}$ & LYPUSR & 4 & 0 & - & - & - & - & - & - & - \\
\hline 18 & $\begin{array}{l}\text { Lyngbya scottii f. minor } \\
\text { (F.E. Fritsch) Elenk. }\end{array}$ & LYSCOR & 3 & 0 & - & - & - & - & - & - & - \\
\hline 19 & Lyngbya sp. & LY001Y & 0 & $1-4$ & - & - & - & - & - & - & - \\
\hline 20 & $\begin{array}{l}\text { Merismopedia glauca } \\
\text { (Ehrb.) Kütz. }\end{array}$ & MRG01Y & 1 & 0 & P-B & - & - & - & o-a & $\mathrm{i}$ & ind \\
\hline 21 & $\begin{array}{l}\text { Merismopedia punctata } \\
\text { Meyen }\end{array}$ & MPUNOA & 3 & 0 & P-B & - & - & - & o-a & $\mathrm{i}$ & ind \\
\hline 22 & $\begin{array}{l}\text { Merismopedia tenuissima } \\
\text { Lemm. }\end{array}$ & MRT01Y & 0 & $2-6$ & P-B & - & - & - & b-a & hl & - \\
\hline 23 & $\begin{array}{l}\text { Microcystis aeruginosa } \\
\text { (Kütz.) Kütz. }\end{array}$ & MIA01Y & 0 & $2-6$ & $\mathrm{P}$ & - & - & - & o-a & hl & - \\
\hline 24 & $\begin{array}{l}\text { Oscillatoria animalis } \\
\text { Gom. }\end{array}$ & OSAN1Y & 0 & 3 & P-B & - & str & - & 0 & - & - \\
\hline 25 & Oscillatoria brevis Gom. & OSB01Y & 0 & $1-4$ & P-B & - & st & - & b-p & - & - \\
\hline 26 & $\begin{array}{l}\text { Oscillatoria formosa } \\
\text { Gom. }\end{array}$ & OSFO0A & 0 & 1 & P-B & - & st & - & b-p & - & - \\
\hline 27 & $\begin{array}{l}\text { Oscillatoria guttulata } \\
\text { Van Goor }\end{array}$ & OSGU1Y & 0 & 2 & $\mathrm{P}$ & - & st & - & $\mathrm{a}$ & - & - \\
\hline 28 & $\begin{array}{l}\text { Oscillatoria mougeotii } \\
\text { Kütz. }\end{array}$ & OSCMOQ & 0 & 1 & P-B & - & st & - & $\mathrm{o}-\mathrm{a}$ & - & - \\
\hline 29 & $\begin{array}{l}\text { Oscillatoria princeps } \\
\text { Gom. }\end{array}$ & OSP01Y & 0 & 1 & P-B & - & $\begin{array}{l}\text { st- } \\
\text { str }\end{array}$ & - & b-p & - & - \\
\hline 30 & Oscillatoria sp. & OS001Y & 0 & $1-6$ & - & - & - & - & - & - & - \\
\hline 31 & $\begin{array}{l}\text { Phormidium ambiguum } \\
\text { Gom. }\end{array}$ & PRA01Y & 5 & 0 & B & temp & $\begin{array}{l}\text { st- } \\
\text { str }\end{array}$ & - & $\mathrm{b}$ & $\mathrm{i}$ & ind \\
\hline 32 & $\begin{array}{l}\text { Phormidium autumnale } \\
\text { (Ag.) Gom. }\end{array}$ & PRAU1Y & $1-6$ & 0 & B & - & $\begin{array}{l}\text { st- } \\
\text { str }\end{array}$ & - & $\mathrm{b}$ & - & - \\
\hline 33 & $\begin{array}{l}\text { Phormidium foveolarum } \\
\text { Gom. }\end{array}$ & PHOFOQ & 0 & 2 & B & - & - & - & b-o & - & - \\
\hline 34 & Phormidium sp. & PR001Y & 0 & $1-3$ & - & - & - & - & - & - & - \\
\hline 35 & $\begin{array}{l}\text { Phormidium subfuscum } \\
\text { Gom. }\end{array}$ & PHORSJ & 6 & 0 & B & - & $\begin{array}{l}\text { st- } \\
\text { str }\end{array}$ & - & $\mathrm{b}$ & - & - \\
\hline 36 & $\begin{array}{l}\text { Phormidium uncinatum } \\
\text { Gom. }\end{array}$ & PRU01Y & $1-5$ & 0 & P-B & temp & - & - & $\mathrm{b}$ & $\mathrm{i}$ & - \\
\hline 37 & $\begin{array}{l}\text { Planktolyngbya regularis } \\
\text { Kom.-Legn. et Tavera }\end{array}$ & PLR01Y & 0 & $2-3$ & $\mathrm{P}$ & warm & st & - & - & - & - \\
\hline 38 & $\begin{array}{l}\text { Pleurocapsa crepidinum } \\
\text { Collins }\end{array}$ & PCC01Y & 0 & 4 & Ep & - & - & - & - & $\mathrm{ph}$ & - \\
\hline 39 & $\begin{array}{l}\text { Rhabdogloea smithii (R. } \\
\text { et F. Chodat) Komárek }\end{array}$ & DACTRR & 1 & 0 & $\mathrm{P}$ & - & $\begin{array}{l}\text { st- } \\
\text { str }\end{array}$ & - & - & - & - \\
\hline 40 & $\begin{array}{l}\text { Schizothrix pulvinata } \\
\text { (Kütz.) Gom. }\end{array}$ & SZP01Y & 0 & 2 & Ep & - & $\begin{array}{l}\text { st- } \\
\text { str }\end{array}$ & - & - & & - \\
\hline 41 & Spirulina major Kütz. & SPM01Y & 0 & $2-4$ & $\mathrm{P}$ & - & st & - & - & $\mathrm{ph}$ & - \\
\hline 42 & $\begin{array}{l}\text { Spirulina platensis } \\
\text { (Nordst. ex Gom.) Geitler }\end{array}$ & SPP01Y & 0 & 2 & $\mathrm{P}$ & - & st & - & $\mathrm{b}$ & - & - \\
\hline 43 & Spirulina sp. & SPIRUQ & 0 & 1 & - & - & - & - & - & - & - \\
\hline
\end{tabular}




\begin{tabular}{|c|c|c|c|c|c|c|c|c|c|c|c|}
\hline No & Taxon & Code & Rud & Qish & Hab & $\mathbf{T}$ & Oxy & D & $\mathbf{S}$ & Hal & pH \\
\hline 44 & $\begin{array}{l}\text { Xenococcus pallidus } \\
\text { (Hansg.) Komarek et } \\
\text { Anagn. } \\
\text { Chrysophyta }\end{array}$ & XENCPQ & 0 & $1-2$ & Ep & - & - & - & - & $\mathrm{ph}$ & - \\
\hline 45 & $\begin{array}{l}\text { Hydrurus foetidus } \\
\text { (Villars) Trevisan }\end{array}$ & HYDFOL & $2-6$ & 0 & B & - & - & - & $\mathrm{o}-\mathrm{x}$ & - & - \\
\hline 46 & Lagynion janei Bourr. & LAGYJQ & 0 & 6 & Ep & - & - & - & - & - & - \\
\hline 47 & $\begin{array}{c}\text { Stylococcus aureus Chod. } \\
\text { Xanthophyta }\end{array}$ & SYA01Y & 0 & $2-4$ & Ep & - & - & - & - & - & - \\
\hline 48 & $\begin{array}{l}\text { Characiopsis } \\
\text { microcysticola Skuja }\end{array}$ & CHAMIR & 2 & 0 & - & - & - & - & - & - & - \\
\hline 49 & $\begin{array}{l}\text { Gloeobotrys } \\
\text { monochloron Ettl }\end{array}$ & GLOEBR & 6 & 0 & - & - & - & - & - & - & - \\
\hline 50 & $\begin{array}{l}\text { Heterothrix monochloron } \\
\text { Ettl }\end{array}$ & HETERR & $1-2$ & 0 & - & - & - & - & - & - & - \\
\hline 51 & $\begin{array}{l}\text { Tribonema subtilissimum } \\
\text { Pasch. }\end{array}$ & TRISUR & 4 & 0 & B & - & - & - & - & $\mathrm{i}$ & - \\
\hline 52 & $\begin{array}{l}\text { Tribonema vulgare } \\
\text { Pasch. } \\
\text { Cryptophyta }\end{array}$ & TRIVUR & 2 & 0 & P-B & - & - & - & $\mathrm{o}-\mathrm{a}$ & $\mathrm{i}$ & - \\
\hline 53 & $\begin{array}{l}\text { Cryptomonas sp. } \\
\text { Euglenophyta }\end{array}$ & CRYPTQ & 0 & 1 & - & - & - & - & - & - & - \\
\hline 54 & Euglena acus Ehrb. & EUA01Y & 0 & $1-2$ & $P$ & eterm & st & - & $\mathrm{b}$ & $\mathrm{i}$ & ind \\
\hline 55 & $\begin{array}{l}\text { Euglena ehrenbergii } \\
\text { Klebs }\end{array}$ & EUE01Y & 0 & 1 & P-B & eterm & $\begin{array}{l}\text { st- } \\
\text { str }\end{array}$ & - & b & - & ind \\
\hline 56 & $\begin{array}{l}\text { Euglena oxyuris } \\
\text { Schmarda }\end{array}$ & EUOXYL & 3 & 0 & $\mathrm{P}$ & & & - & b-a & - & - \\
\hline 57 & Euglena proxima Dang. & EUP01Y & 0 & 1 & P-B & eterm & $\begin{array}{l}\text { st- } \\
\text { str }\end{array}$ & - & $\mathrm{p}$ & $\mathrm{mh}$ & ind \\
\hline 58 & Euglena sp. & EU001Y & 0 & 1 & - & - & - & - & - & - & - \\
\hline 59 & $\begin{array}{l}\text { Euglena texta (Duj.) } \\
\text { Hübn. }\end{array}$ & EUT01Y & 0 & $1-3$ & $\mathrm{P}$ & eterm & $\begin{array}{l}\text { st- } \\
\text { str }\end{array}$ & - & $\mathrm{b}$ & - & ind \\
\hline 60 & $\begin{array}{l}\text { Petalomonas } \\
\text { mediocanellata Stein }\end{array}$ & PETALR & 1 & 0 & B & warm & $\begin{array}{l}\text { st- } \\
\text { str }\end{array}$ & - & $\mathrm{a}$ & - & ind \\
\hline 61 & $\begin{array}{l}\text { Phacus acuminatus } \\
\text { Stokes }\end{array}$ & PHACAQ & 0 & 1 & P-B & eterm & $\begin{array}{l}\text { st- } \\
\text { str }\end{array}$ & - & b-a & $\mathrm{i}$ & - \\
\hline 62 & Phacus parvulus Klebs & PHAPAQ & 0 & 5 & $\mathrm{P}$ & eterm & $\begin{array}{l}\text { st- } \\
\text { str }\end{array}$ & - & $\mathrm{b}$ & $\mathrm{i}$ & ind \\
\hline 63 & $\begin{array}{l}\text { Phacus pleuronectens } \\
\text { (Ehrb.) Duj. }\end{array}$ & PHP01Y & 0 & 1 & P-B & - & $\begin{array}{l}\text { st- } \\
\text { str }\end{array}$ & - & b-a & $\mathrm{i}$ & ind \\
\hline 64 & $\begin{array}{l}\text { Phacus pyrum (Ehrb.) } \\
\text { Stein }\end{array}$ & PHPY1Y & 0 & 1 & $\mathrm{P}$ & eterm & $\begin{array}{l}\text { st- } \\
\text { str }\end{array}$ & - & $\mathrm{b}$ & $\mathrm{i}$ & ind \\
\hline 65 & Phacus sp. & PHACUH & 0 & 1 & - & - & - & - & - & - & - \\
\hline 66 & Phacus striatus Francé & PHASTQ & 0 & 1 & P-B & eterm & $\begin{array}{l}\text { st- } \\
\text { str }\end{array}$ & - & b-a & - & ind \\
\hline 67 & $\begin{array}{l}\text { Trachelomonas hispida } \\
\text { Delf. } \\
\quad \text { Bacillariophyta }\end{array}$ & TRH01Y & 1 & 0 & P-B & eterm & $\begin{array}{l}\text { st- } \\
\text { str }\end{array}$ & & $\mathrm{b}$ & $\mathrm{i}$ & \\
\hline 68 & $\begin{array}{l}\text { Achnanthes chlidanos } \\
\text { Hohn et Hellerman }\end{array}$ & AC9960 & 4 & 0 & B & - & - & $\mathrm{sx}$ & o & $\mathrm{i}$ & ind \\
\hline 69 & $\begin{array}{l}\text { Achnanthes delicatula } \\
\text { subsp. hauckiana (Grun. } \\
\text { in Cleve et Grun.) Lange- } \\
\text { Bert. et Ruppel }\end{array}$ & ACDE1R & 1 & 0 & - & - & - & - & - & - & - \\
\hline
\end{tabular}




\begin{tabular}{|c|c|c|c|c|c|c|c|c|c|c|c|}
\hline No & Taxon & Code & Rud & Qish & Hab & $\mathbf{T}$ & Oxy & D & $\mathbf{S}$ & Hal & pH \\
\hline 70 & $\begin{array}{l}\text { Achnanthes lanceolata } \mathrm{f} . \\
\text { capitata } \mathrm{O} \text {. Müll. }\end{array}$ & ACLC1L & 2 & 0 & B & - & - & - & $x-b$ & $\mathrm{i}$ & ind \\
\hline 71 & $\begin{array}{l}\text { Achnanthes lanceolata f. } \\
\text { ventricosa Hust. }\end{array}$ & ACLV1L & $1-2$ & 0 & B & warm & - & sx & $x-b$ & $\mathrm{i}$ & alf \\
\hline 72 & $\begin{array}{l}\text { Achnanthes lanceolata } \\
\text { (Bréb.) Grun. var. } \\
\text { lanceolata }\end{array}$ & AL001Y & $1-3$ & 0 & P-B & warm & $\begin{array}{l}\text { st- } \\
\text { str }\end{array}$ & sx & $\mathrm{o}-\mathrm{x}$ & $\mathrm{i}$ & alf \\
\hline 73 & $\begin{array}{l}\text { Achnanthes lineariformis } \\
\text { Lange-Bert. }\end{array}$ & AC9959 & $3-6$ & 1 & B & eterm & $\begin{array}{l}\text { st- } \\
\text { str }\end{array}$ & es & $\mathrm{x}-\mathrm{O}$ & $\mathrm{i}$ & ind \\
\hline 74 & $\begin{array}{l}\text { Actinocyclus normanii } \\
\text { (Greg. ex Grev.) Hust. ex } \\
\text { Van Landingham }\end{array}$ & ACTINQ & 0 & 1 & $\mathrm{P}$ & - & $\begin{array}{l}\text { st- } \\
\text { str }\end{array}$ & - & - & $\mathrm{mh}$ & - \\
\hline 75 & $\begin{array}{l}\text { Amphora coffeaeformis } \\
\text { (Ag.) Kütz. }\end{array}$ & AMC01Y & 0 & $1-6$ & B & - & $\begin{array}{l}\text { st- } \\
\text { str }\end{array}$ & - & - & $\mathrm{mh}$ & - \\
\hline 76 & $\begin{array}{l}\text { Amphora montana } \\
\text { Krasske }\end{array}$ & AMMONO & 0 & 1 & B & - & ae & - & - & $\mathrm{i}$ & alf \\
\hline 77 & $\begin{array}{l}\text { Amphora ovalis (Kütz.) } \\
\text { Kütz. }\end{array}$ & AMO01Y & $1-2$ & 1 & B & temp & $\begin{array}{l}\text { st- } \\
\text { str }\end{array}$ & sx & $a-b$ & $\mathrm{i}$ & alf \\
\hline 78 & $\begin{array}{l}\text { Amphora pediculus } \\
\text { (Kütz.) Grun. }\end{array}$ & AMP01Y & 1 & 1 & B & temp & st & es & o-a & $\mathrm{i}$ & alf \\
\hline 79 & Amphora veneta Kütz. & AM004A & 0 & $1-2$ & B & - & - & es & o & $\mathrm{i}$ & alf \\
\hline 80 & $\begin{array}{l}\text { Anomoeoneis } \\
\text { sphaerophora (Ehrb.) } \\
\text { Pfitz. }\end{array}$ & AN009A & 0 & $1-2$ & P-B & warm & $\begin{array}{l}\text { st- } \\
\text { str }\end{array}$ & - & $x-b$ & hl & alb \\
\hline 81 & $\begin{array}{l}\text { Anomoeoneis } \\
\text { sphaerophora f. sculpta } \\
\text { Kramm. }\end{array}$ & ANOSSQ & 0 & 1 & B & - & - & - & - & $\mathrm{mh}$ & - \\
\hline 82 & $\begin{array}{l}\text { Aulacoseira granulata } \\
\text { (Ehrb.) Simons. }\end{array}$ & AUL01Y & 1 & $1-3$ & P-B & temp & $\begin{array}{l}\text { st- } \\
\text { str }\end{array}$ & es & b-a & $\mathrm{i}$ & alf \\
\hline 83 & $\begin{array}{l}\text { Bacillaria paxillifer (O. } \\
\text { Müll.) Hendey }\end{array}$ & BAP01Y & 0 & $1-4$ & P-B & - & - & es & o & $\mathrm{mh}$ & ind \\
\hline 84 & $\begin{array}{l}\text { Caloneis leptosoma } \\
\text { (Grun.) Kramm. in } \\
\text { Kramm. et Lange-Bert. }\end{array}$ & CALLER & 1 & 0 & B & - & - & - & o & - & - \\
\hline 85 & $\begin{array}{l}\text { Caloneis molaris (Grun.) } \\
\text { Kramm. }\end{array}$ & CALMOR & 1 & 0 & B & - & str & es & - & $\mathrm{i}$ & ind \\
\hline 86 & $\begin{array}{l}\text { Caloneis permagna (J.W. } \\
\text { Bailey) Cleve }\end{array}$ & CLP01Y & 1 & $1-2$ & B & - & - & - & - & hl & - \\
\hline 87 & $\begin{array}{l}\text { Caloneis silicula (Ehrb.) } \\
\text { Cl. var. silicula }\end{array}$ & CASILR & 1 & 0 & - & - & - & - & - & - & - \\
\hline 88 & $\begin{array}{l}\text { Caloneis silicula var. } \\
\text { truncatula (Grun.) } \mathrm{Cl} \text {. }\end{array}$ & CALSTL & 1 & 0 & B & - & st & - & - & $\mathrm{i}$ & alf \\
\hline 89 & $\begin{array}{l}\text { Caloneis silicula var. } \\
\text { ventricosa (Ehrb.) Donk. }\end{array}$ & CALSVL & 1 & 0 & B & - & st & - & - & $\mathrm{i}$ & - \\
\hline 90 & $\begin{array}{l}\text { Campylodiscus bicostatus } \\
\text { W. Smith in Roper }\end{array}$ & СМРCBH & 0 & 1 & B & - & - & - & - & $\mathrm{mh}$ & - \\
\hline 91 & $\begin{array}{l}\text { Cocconeis placentula var. } \\
\text { euglypta (Ehrb.) Grun. }\end{array}$ & CCCPEQ & $1-4$ & 1 & P-B & temp & $\begin{array}{l}\text { st- } \\
\text { str }\end{array}$ & sx & - & $\mathrm{i}$ & alf \\
\hline 92 & $\begin{array}{l}\text { Cocconeis placentula } \\
\text { Ehrb. var. placentula }\end{array}$ & ССР01Y & $1-3$ & 0 & P-B & temp & $\begin{array}{l}\text { st- } \\
\text { str }\end{array}$ & es & $o-b$ & $\mathrm{i}$ & alf \\
\hline 93 & $\begin{array}{l}\text { Cocconeis placentula var. } \\
\text { pseudolineata Geitl. }\end{array}$ & CCCPPQ & 0 & 1 & $\mathrm{P}$ & - & $\begin{array}{l}\text { st- } \\
\text { str }\end{array}$ & sx & - & $\mathrm{i}$ & alf \\
\hline 94 & $\begin{array}{l}\text { Craticula accomoda } \\
\text { (Hust.) D.G. Mann }\end{array}$ & CRA01Y & 0 & 1 & $\mathrm{P}$ & - & - & $\mathrm{sp}$ & o-a & $\mathrm{i}$ & \\
\hline 95 & $\begin{array}{l}\text { Craticula ambigua } \\
\text { (Ehrb.) Mann }\end{array}$ & CRA00A & 1 & 0 & B & warm & st & es & o & $\mathrm{i}$ & alf \\
\hline
\end{tabular}




\begin{tabular}{|c|c|c|c|c|c|c|c|c|c|c|c|}
\hline No & $\begin{array}{c}\text { Taxon } \\
\end{array}$ & Code & Rud & Qish & Hab & $\mathbf{T}$ & Oxy & D & $\mathbf{S}$ & Hal & $\mathbf{p H}$ \\
\hline 96 & $\begin{array}{l}\text { Craticula cuspidata } \\
\text { (Kütz.) D.G.Mann }\end{array}$ & CRC01Y & 1 & 1 & $\mathrm{~B}$ & temp & st & es & o & $\mathrm{i}$ & alf \\
\hline 97 & $\begin{array}{l}\text { Craticula halophila } \\
\text { (Grun. ex Van Heurck) } \\
\text { Mann }\end{array}$ & CRATGO & 0 & 1 & B & - & $\begin{array}{l}\text { st- } \\
\text { str }\end{array}$ & es & - & $\mathrm{mh}$ & alf \\
\hline 98 & $\begin{array}{l}\text { Cyclotella meneghiniana } \\
\text { Kütz. }\end{array}$ & CYM01Y & 1 & $1-6$ & P-B & temp & st & $\mathrm{sp}$ & $\mathrm{o}-\mathrm{a}$ & $\mathrm{hl}$ & alf \\
\hline 99 & $\begin{array}{l}\text { Cyclotella stelligera } \\
\text { (Cleve et Grun. in Cleve) } \\
\text { Van Heurck }\end{array}$ & CYSTER & 1 & 0 & P-B & - & st & es & $\mathrm{x}$ & $\mathrm{i}$ & ind \\
\hline 100 & $\begin{array}{l}\text { Cyclotella tuberculata } \\
\text { Makar. et Log. }\end{array}$ & CYCTUQ & 0 & 1 & $\mathrm{P}$ & - & - & - & - & hl & - \\
\hline 101 & $\begin{array}{l}\text { Cylindrotheca gracilis } \\
\text { (Bréb. ex Kütz.) Grun. in } \\
\text { Van Heurck }\end{array}$ & CYLIGQ & 0 & 2 & B & - & st & - & o & $\mathrm{hl}$ & - \\
\hline 102 & $\begin{array}{l}\text { Cymatopleura librile } \\
\text { (Ehrb.) Pant. }\end{array}$ & CL001A & $1-3$ & 0 & P-B & - & - & - & $\mathrm{o}$ & $\mathrm{i}$ & alf \\
\hline 103 & Cymbella affinis Kütz. & CYAFFR & 2 & 0 & B & temp & $\begin{array}{l}\text { st- } \\
\text { str }\end{array}$ & $\mathrm{sx}$ & b-o & $\mathrm{i}$ & alf \\
\hline 104 & $\begin{array}{l}\text { Cymbella aspera (Ehrb.) } \\
\text { Perag. in Pelletan }\end{array}$ & CYASPR & 2 & 0 & B & - & $\begin{array}{l}\text { st- } \\
\text { str }\end{array}$ & es & b-o & $\mathrm{i}$ & alf \\
\hline 105 & $\begin{array}{l}\text { Cymbella cistula (Ehrb.) } \\
\text { Kirchn. }\end{array}$ & CYCISR & $1-4$ & 0 & B & - & $\begin{array}{l}\text { st- } \\
\text { str }\end{array}$ & sx & $o-b$ & $\mathrm{i}$ & alf \\
\hline 106 & $\begin{array}{l}\text { Cymbella cornuta (Ehrb.) } \\
\text { R. Ross }\end{array}$ & CYLANR & 2 & 0 & B & - & - & sx & o & $\mathrm{i}$ & alf \\
\hline 107 & Cymbella cuspidata Kütz. & CYCUSR & 1 & 0 & B & temp & - & - & $\mathrm{o}-\mathrm{a}$ & $\mathrm{i}$ & ind \\
\hline 108 & $\begin{array}{l}\text { Cymbella ehrenbergii } \\
\text { Kütz. }\end{array}$ & CYEHRR & 1 & 0 & B & - & $\begin{array}{l}\text { st- } \\
\text { str }\end{array}$ & - & b-o & $\mathrm{i}$ & alb \\
\hline 109 & Cymbella hybrida Cleve & CYMHYQ & 0 & 1 & B & - & - & - & - & $\mathrm{hl}$ & alb \\
\hline 110 & $\begin{array}{l}\text { Cymbella microcephala } \\
\text { Grun. in Van Heurck }\end{array}$ & CYMBMO & 0 & $1-2$ & B & - & - & es & $\mathrm{b}$ & $\mathrm{i}$ & alf \\
\hline 111 & $\begin{array}{l}\text { Cymbella naviculiformis } \\
\text { (Auersw. ex Heib.) Cleve }\end{array}$ & CM009A & 1 & 0 & B & - & - & es & o & $\mathrm{i}$ & ind \\
\hline 112 & $\begin{array}{l}\text { Cymbella reinhardtii } \\
\text { Grun. }\end{array}$ & CM099A & 1 & 0 & B & - & - & - & - & - & - \\
\hline 113 & Cymbella sp. & CYMBEH & 0 & 1 & B & - & - & - & - & - & - \\
\hline 114 & $\begin{array}{l}\text { Cymbella subcuspidata } \\
\text { Kramm. }\end{array}$ & CYHETL & 1 & 0 & B & - & - & - & - & $\mathrm{i}$ & acf \\
\hline 115 & $\begin{array}{l}\text { Cymbella tumidula Grun. } \\
\text { in A. Schmidt }\end{array}$ & CM109A & 1 & 0 & B & - & - & - & - & $\mathrm{i}$ & alf \\
\hline 116 & Cymbella turgidula Grun. & CYTURL & 1 & 0 & B & - & $\begin{array}{l}\text { st- } \\
\text { str }\end{array}$ & es & - & - & ind \\
\hline 117 & $\begin{array}{l}\text { Diatoma hiemale (Roth) } \\
\text { Heib. }\end{array}$ & DIHIER & $2-4$ & 0 & P-B & cool & $\begin{array}{l}\text { st- } \\
\text { str }\end{array}$ & sx & b-o & $\mathrm{hb}$ & ind \\
\hline 118 & $\begin{array}{l}\text { Diatoma mesodon (Ehrb.) } \\
\text { Grun. }\end{array}$ & DT021A & $1-6$ & 0 & B & cool & $\begin{array}{l}\text { st- } \\
\text { str }\end{array}$ & sx & $o-b$ & $\mathrm{hb}$ & - \\
\hline 119 & Diatoma tenue Ag. & DITENR & $1-4$ & 0 & P-B & - & st & sx & b-a & $\mathrm{hl}$ & ind \\
\hline 120 & Diatoma vulgare Bory & DIVULR & $1-3$ & 0 & P-B & - & st- & sx & b-a & $\mathrm{i}$ & ind \\
\hline 121 & $\begin{array}{l}\text { Didymosphenia geminata } \\
\text { (Lyngb.) M. Schm. in A. } \\
\text { Schmidt }\end{array}$ & DYDGER & 1 & 0 & B & - & $\begin{array}{l}\text { st- } \\
\text { str }\end{array}$ & sx & $\mathrm{o}-\mathrm{a}$ & $\mathrm{i}$ & ind \\
\hline 122 & $\begin{array}{l}\text { Diploneis ovalis (Hilse) } \\
\text { Cleve }\end{array}$ & DIPLOR & 1 & 0 & B & - & - & $\mathrm{sp}$ & $\mathrm{b}$ & $\mathrm{i}$ & alb \\
\hline 123 & $\begin{array}{l}\text { Encyonema elginense } \\
\text { (Kramm.) D.G. Mann }\end{array}$ & ENCELQ & 0 & 2 & B & - & st & sx & - & $\mathrm{hb}$ & acf \\
\hline
\end{tabular}




\begin{tabular}{|c|c|c|c|c|c|c|c|c|c|c|c|}
\hline No & Taxon & Code & Rud & Qish & Hab & $\mathbf{T}$ & Oxy & D & $\mathbf{S}$ & Hal & pH \\
\hline 124 & $\begin{array}{l}\text { Encyonema minutum } \\
\text { (Hilse ex Rabenh.) D.G. } \\
\text { Mann }\end{array}$ & ENCMML & 1 & 0 & $\mathrm{~B}$ & - & - & es & o-b & oh & ind \\
\hline 125 & $\begin{array}{l}\text { Encyonema silesiacum } \\
\text { (Bleisch in Rabenh.) } \\
\text { Mann }\end{array}$ & ENCSIQ & 1 & 3 & B & - & $\begin{array}{l}\text { st- } \\
\text { str }\end{array}$ & sx & $\mathrm{x}-\mathrm{O}$ & $\mathrm{i}$ & ind \\
\hline 126 & $\begin{array}{l}\text { Entomoneis alata (Ehrb.) } \\
\text { Ehrb. }\end{array}$ & ENO01Y & 0 & $1-3$ & P-B & - & st & - & - & $\mathrm{mh}$ & alf \\
\hline 127 & $\begin{array}{l}\text { Entomoneis paludosa (W. } \\
\text { Sm.) Reim. }\end{array}$ & ENP01Y & 0 & $1-3$ & B & - & - & - & - & - & - \\
\hline 128 & $\begin{array}{l}\text { Epithemia adnata var. } \\
\text { porcellus (Kütz.) R. Ross }\end{array}$ & EPIAPR & 1 & 0 & B & - & - & - & $\mathrm{b}$ & $\mathrm{i}$ & alb \\
\hline 129 & $\begin{array}{l}\text { Eunotia bilunaris (Ehrb.) } \\
\text { Mills }\end{array}$ & EUNBIR & 1 & 0 & B & temp & - & es & $\mathrm{b}$ & $\mathrm{i}$ & acf \\
\hline 130 & Eunotia diodon Ehrb. & EUNDIR & 1 & 0 & B & cool & st & - & $0-x$ & $\mathrm{i}$ & acf \\
\hline 131 & $\begin{array}{l}\text { Eunotia exigua (Breb ex } \\
\text { Kütz.) Rabenh. }\end{array}$ & EUNGRR & 1 & 0 & B & - & - & - & $\mathrm{a}$ & $\mathrm{hb}$ & ind \\
\hline 132 & $\begin{array}{l}\text { Eunotia minor (Kütz.) } \\
\text { Grun. }\end{array}$ & EU110A & 1 & 0 & B & - & - & es & $\mathrm{x}$ & - & - \\
\hline 133 & Eunotia praerupta Ehrb. & EUNPPR & 1 & 0 & B & cool & $\begin{array}{l}\text { st- } \\
\text { str }\end{array}$ & sx & $\mathrm{b}$ & $\mathrm{hb}$ & acf \\
\hline 134 & $\begin{array}{l}\text { Eunotia steineckei } \\
\text { Petersen }\end{array}$ & EUNEXR & 1 & 0 & B & - & - & es & $o-b$ & $\mathrm{hb}$ & acf \\
\hline 135 & $\begin{array}{l}\text { Fallacia pygmaea (Kütz.) } \\
\text { Stickle et Mann }\end{array}$ & FP001Y & 0 & $1-4$ & B & - & - & es & b-o & $\mathrm{mh}$ & alf \\
\hline 136 & $\begin{array}{l}\text { Fallacia subhamulata } \\
\text { (Grun.) Mann }\end{array}$ & FS001Y & 1 & 0 & B & - & - & - & - & $\mathrm{i}$ & ind \\
\hline 137 & $\begin{array}{l}\text { Fragilaria capucina } \\
\text { Desm. var. capucina }\end{array}$ & FRACAQ & 3 & 3 & B & - & & es & o & $\mathrm{i}$ & alf \\
\hline 138 & $\begin{array}{l}\text { Fragilaria capucina var. } \\
\text { rumpens (Kütz.) Lange- } \\
\text { Bert. }\end{array}$ & FR009G & $1-5$ & 0 & B & - & $\begin{array}{l}\text { st- } \\
\text { str }\end{array}$ & es & o & $\mathrm{i}$ & acf \\
\hline 139 & $\begin{array}{l}\text { Fragilaria capucina var. } \\
\text { vaucheriae (Kütz.) } \\
\text { Lange-Bert. }\end{array}$ & FRAVCR & $3-4$ & 0 & $\mathrm{P}$ & - & - & sx & $o-b$ & $\mathrm{i}$ & alf \\
\hline 140 & $\begin{array}{l}\text { Fragilaria construens } \mathrm{f} \text {. } \\
\text { construens (Ehrb.) Hust. }\end{array}$ & STRSCQ & 0 & 1 & P-B & temp & $\begin{array}{l}\text { st- } \\
\text { str }\end{array}$ & sx & o & $\mathrm{i}$ & alf \\
\hline 141 & $\begin{array}{l}\text { Fragilaria crotonensis } \\
\text { Kitt. }\end{array}$ & FRACRR & 1 & 0 & $\mathrm{P}$ & - & st & es & $a-b$ & hl & alf \\
\hline 142 & $\begin{array}{l}\text { Fragilaria fasciculata } \\
\text { (Ag.) Lange-Bert. }\end{array}$ & FR057A & 0 & $1-2$ & B & - & st & sx & o & $\mathrm{hl}$ & alf \\
\hline 143 & $\begin{array}{l}\text { Fragilaria pulchella } \\
\text { (Ralfs ex Kütz.) Lange- } \\
\text { Bert. }\end{array}$ & CTEKRL & 2 & 0 & Ep & - & - & - & b & $\mathrm{hl}$ & - \\
\hline 144 & $\begin{array}{l}\text { Fragilaria ulna var. acus } \\
\text { (Kütz.) Lange-Bert. }\end{array}$ & SYNACR & 1 & 0 & $\mathrm{P}$ & - & $\begin{array}{l}\text { st- } \\
\text { str }\end{array}$ & es & $\mathrm{b}$ & $\mathrm{i}$ & alb \\
\hline 145 & $\begin{array}{l}\text { Fragilaria vaucheriae } \\
\text { (Kütz.) Peters. }\end{array}$ & FRAVVR & $1-4$ & 0 & $\mathrm{P}$ & - & - & $\mathrm{sx}$ & $o-b$ & $\mathrm{i}$ & alf \\
\hline 146 & $\begin{array}{l}\text { Fragilariforma bicapitata } \\
\text { (Mayer) Will. et Round }\end{array}$ & FRFOBR & 1 & 0 & B & - & - & - & o-b & $\mathrm{hb}$ & ind \\
\hline 147 & $\begin{array}{l}\text { Fragilariforma virescens } \\
\text { (Ralfs) Williams et } \\
\text { Round }\end{array}$ & FRFVIR & 1 & 0 & P-B & - & st & es & o & $\mathrm{i}$ & ind \\
\hline 148 & $\begin{array}{l}\text { Frustulia rhomboides } \\
\text { (Ehrb.) De Toni }\end{array}$ & FRURHR & $1-2$ & 0 & B & - & st & es & $x-b$ & $\mathrm{hb}$ & acf \\
\hline 149 & $\begin{array}{l}\text { Frustulia vulgaris (Thw.) } \\
\text { De Toni }\end{array}$ & FU001A & $1-3$ & 0 & P-B & - & st & es & $x-b$ & $\mathrm{i}$ & alf \\
\hline
\end{tabular}




\begin{tabular}{|c|c|c|c|c|c|c|c|c|c|c|c|}
\hline No & Taxon & Code & Rud & Qish & Hab & $\mathbf{T}$ & Oxy & D & $\mathbf{S}$ & Hal & pH \\
\hline 150 & $\begin{array}{l}\text { Gomphoneis } \\
\text { quadripunctatum (Østr.) } \\
\text { Dawson ex Ross et Sims }\end{array}$ & GOMPQR & $1-2$ & 0 & $\mathrm{~B}$ & - & - & es & - & $\mathrm{i}$ & ind \\
\hline 151 & $\begin{array}{l}\text { Gomphonema } \\
\text { acuminatum Ehrb. var. } \\
\text { acuminatum }\end{array}$ & GOMACR & 1 & 0 & P-B & - & st & es & $x-b$ & $\mathrm{i}$ & alf \\
\hline 152 & $\begin{array}{l}\text { Gomphonema } \\
\text { acuminatum var. } \\
\text { coronatum (Ehrb.) W. } \\
\text { Sm. }\end{array}$ & GOMAAR & 1 & 0 & P-B & - & st & - & b & $\mathrm{i}$ & ind \\
\hline 153 & $\begin{array}{l}\text { Gomphonema affine } \\
\text { Kütz. }\end{array}$ & GO020A & 0 & $1-5$ & P-B & - & st & es & $o-b$ & - & - \\
\hline 154 & $\begin{array}{l}\text { Gomphonema } \\
\text { angustatum (Kütz.) } \\
\text { Rabenh. }\end{array}$ & GOA01Y & $1-4$ & 1 & P-B & - & $\begin{array}{l}\text { st- } \\
\text { str }\end{array}$ & es & $\mathrm{b}$ & $\mathrm{i}$ & alf \\
\hline 155 & $\begin{array}{l}\text { Gomphonema angustum } \\
\text { Ag. }\end{array}$ & GO073A & 0 & 1 & P-B & - & $\begin{array}{l}\text { st- } \\
\text { str }\end{array}$ & es & $o-b$ & $\mathrm{i}$ & ind \\
\hline 156 & $\begin{array}{l}\text { Gomphonema clavatum } \\
\text { E. Reichardt }\end{array}$ & GO029A & 1 & 0 & B & - & str & es & $o-b$ & $\mathrm{i}$ & ind \\
\hline 157 & $\begin{array}{l}\text { Gomphonema minutum } \\
\text { (Ag.) Ag. }\end{array}$ & GOMMIO & 0 & 1 & B & - & - & es & $o-b$ & oh & alf \\
\hline 158 & $\begin{array}{l}\text { Gomphonema parvulum } \\
\text { Kütz. }\end{array}$ & GO013A & $1-3$ & 1 & B & temp & str & es & $\mathrm{x}$ & $\mathrm{i}$ & ind \\
\hline 159 & $\begin{array}{l}\text { Gomphonema productum } \\
\text { (Grun.) Lange-Bert. et E. } \\
\text { Reichardt }\end{array}$ & GO003B & $1-5$ & 0 & B & - & str & es & $\mathrm{b}$ & $\mathrm{i}$ & alf \\
\hline 160 & $\begin{array}{l}\text { Gomphonema truncatum } \\
\text { Ehrb. sensu Patrick et } \\
\text { Reim. }\end{array}$ & GO023A & 1 & 0 & P-B & - & - & es & $0-\mathrm{x}$ & - & - \\
\hline 161 & $\begin{array}{l}\text { Gyrosigma acuminatum } \\
\text { (Kütz.) Rabenh. }\end{array}$ & GY005A & 1 & $1-5$ & B & cool & - & - & $0-\mathrm{x}$ & $\mathrm{i}$ & alf \\
\hline 162 & $\begin{array}{l}\text { Hannaea arcus f. arcus } \\
\text { (Ehrb.) Patr. }\end{array}$ & HANNAR & $1-6$ & 0 & B & - & str & es & $\mathrm{o}$ & $\mathrm{i}$ & alf \\
\hline 163 & $\begin{array}{l}\text { Hannaea arcus f. recta } \\
\text { (Cl.) Foged }\end{array}$ & HANNRR & $1-5$ & 0 & B & - & - & sx & - & - & - \\
\hline 164 & $\begin{array}{l}\text { Hannaea arcus var. } \\
\text { amphioxys (Rabenh.) } \\
\text { Patr. }\end{array}$ & HANAAL & $1-3$ & 0 & B & cool & str & - & $\mathrm{x}$ & $\mathrm{i}$ & alf \\
\hline 165 & $\begin{array}{l}\text { Hantzschia amphioxys } \\
\text { (Ehrb.) Grun. in Cl. et } \\
\text { Grun. }\end{array}$ & HA001A & $1-2$ & 1 & B & temp & - & es & b-o & $\mathrm{i}$ & ind \\
\hline 166 & Licmophora sp. & LICMOQ & 0 & $1-2$ & - & - & - & - & - & - & - \\
\hline 167 & $\begin{array}{l}\text { Luticola mutica (Kütz.) } \\
\text { Mann }\end{array}$ & LUM01Y & $1-2$ & 1 & B & - & $\begin{array}{l}\text { st- } \\
\text { str }\end{array}$ & $\mathrm{sp}$ & o & $\mathrm{i}$ & ind \\
\hline 169 & $\begin{array}{l}\text { Luticola muticopsis (Van } \\
\text { Heurck) D.G. Mann }\end{array}$ & LUS01Y & 0 & 1 & B & - & $\begin{array}{l}\text { st- } \\
\text { str }\end{array}$ & - & - & - & - \\
\hline 189 & Melosira varians Ag. & ME015A & 2 & 0 & P-B & temp & $\begin{array}{l}\text { st- } \\
\text { str }\end{array}$ & es & $a-b$ & hl & alf \\
\hline 170 & $\begin{array}{l}\text { Meridion circulare } \\
\text { (Grev.) Ag. var. circulare }\end{array}$ & MECIOA & $1-6$ & 0 & B & - & str & es & $o-b$ & $\mathrm{i}$ & alf \\
\hline 171 & $\begin{array}{l}\text { Meridion circulare var. } \\
\text { constrictum (Ralfs) Van } \\
\text { Heurck }\end{array}$ & MECIRR & $1-2$ & 0 & P-B & - & $\begin{array}{l}\text { st- } \\
\text { str }\end{array}$ & sx & $\mathrm{x}$ & $\mathrm{hb}$ & - \\
\hline 172 & Navicula angusta Grun. & NA037A & 0 & 1 & B & - & - & $\mathrm{sx}$ & & hl & acf \\
\hline 173 & $\begin{array}{l}\text { Navicula capitata Ehrb. } \\
\text { var. capitata }\end{array}$ & NACAPR & 1 & 0 & B & temp & - & es & $o-b$ & hl & alf \\
\hline
\end{tabular}




\begin{tabular}{|c|c|c|c|c|c|c|c|c|c|c|c|}
\hline No & Taxon & Code & Rud & Qish & Hab & $\mathbf{T}$ & Oxy & D & $\mathbf{S}$ & Hal & pH \\
\hline 174 & $\begin{array}{l}\text { Navicula capitata var. } \\
\text { hungarica (Grun.) Ross }\end{array}$ & NACUSR & 1 & 0 & B & - & - & es & o & $\mathrm{i}$ & - \\
\hline 175 & $\begin{array}{l}\text { Navicula crucicula (W. } \\
\text { Sm.) Donk. }\end{array}$ & NACRUR & 1 & 0 & B & - & - & & & $\mathrm{mh}$ & ind \\
\hline 176 & $\begin{array}{l}\text { Navicula erifuga Lange- } \\
\text { Bert. }\end{array}$ & NAE01Y & 0 & $1-5$ & B & - & - & es & $\mathrm{x}-\mathrm{O}$ & & \\
\hline 177 & $\begin{array}{l}\text { Navicula gregaria } \\
\text { Donkin }\end{array}$ & NAG01Y & $1-4$ & 1 & P-B & temp & - & es & $x-b$ & $\mathrm{mh}$ & alf \\
\hline 178 & $\begin{array}{l}\text { Navicula peregrina } \\
\text { (Ehrb.) Kütz. }\end{array}$ & NAPERR & 1 & 0 & B & - & - & es & - & $\mathrm{mh}$ & alf \\
\hline 179 & $\begin{array}{l}\text { Navicula perminuta } \\
\text { Grun. in Van Heurck }\end{array}$ & NAPERQ & 0 & $1-6$ & B & - & - & es & - & hl & \\
\hline 180 & Navicula radiosa Kütz. & NA003A & 1 & 0 & B & temp & $\begin{array}{l}\text { st- } \\
\text { str }\end{array}$ & es & $\mathrm{o}$ & $\mathrm{i}$ & ind \\
\hline 181 & $\begin{array}{l}\text { Navicula recens (Lange- } \\
\text { Bert.) Lange-Bert. }\end{array}$ & NAR01Y & 0 & $1-5$ & P-B & - & - & es & o-b & $\mathrm{i}$ & - \\
\hline 182 & $\begin{array}{l}\text { Navicula schroeterii } \\
\text { Meist. }\end{array}$ & NAS01Y & 0 & 1 & B & - & - & es & $a-b$ & $\mathrm{i}$ & alf \\
\hline 183 & Navicula sp. & NA001Y & 0 & 1 & - & - & - & - & - & - & - \\
\hline 184 & Navicula spicula Hickie & NASPIQ & 0 & $1-6$ & P-B & - & - & - & - & $\mathrm{mh}$ & - \\
\hline 185 & Navicula stroemii Hust. & NA650A & 0 & 1 & B & eterm & - & es & o & oh & alf \\
\hline 186 & $\begin{array}{l}\text { Navicula tripunctata (O. } \\
\text { Müll.) Bory }\end{array}$ & NATRIO & 0 & 2 & B & - & $\begin{array}{l}\text { st- } \\
\text { str }\end{array}$ & es & $\mathrm{b}$ & $\mathrm{i}$ & ind \\
\hline 187 & $\begin{array}{l}\text { Navicula trivialis Lange- } \\
\text { Bert. }\end{array}$ & NATRVO & 0 & $1-3$ & B & - & - & $\mathrm{sp}$ & b-o & $\mathrm{i}$ & alf \\
\hline 188 & Navicula veneta Kütz. & NA054A & 0 & $1-6$ & B & - & - & es & $\mathrm{x}-\mathrm{O}$ & hl & alf \\
\hline 189 & $\begin{array}{l}\text { Navicula viridula (Kütz.) } \\
\text { Ehrb. }\end{array}$ & NAV01Y & 1 & 0 & B & - & & es & o & hl & alf \\
\hline 190 & $\begin{array}{l}\text { Naviculadicta protracta } \\
\text { Grun. }\end{array}$ & NADICR & 1 & 0 & B & - & - & es & $x-b$ & $\mathrm{mh}$ & ind \\
\hline 191 & $\begin{array}{l}\text { Neidium affine (Ehrb.) } \\
\text { Cl. }\end{array}$ & NE003A & $2-3$ & 0 & B & - & - & - & o & $\mathrm{i}$ & alf \\
\hline 192 & $\begin{array}{l}\text { Neidium ampliatum } \\
\text { (Ehrb.) Kramm. }\end{array}$ & NEAMPO & 1 & 0 & B & - & st & es & & $\mathrm{hb}$ & ind \\
\hline 193 & $\begin{array}{l}\text { Neidium bisulcatum } \\
\text { (Lagerst.) Cleve }\end{array}$ & NEBISR & 1 & 0 & B & - & - & es & $\mathrm{o}-\mathrm{b}$ & $\mathrm{hb}$ & alf \\
\hline 194 & $\begin{array}{l}\text { Neidium iridis var. } \\
\text { diminutum (Pant.) } \\
\text { Wislouch et Kolbe }\end{array}$ & NEIRDR & 1 & 0 & B & - & - & - & $\mathrm{o}$ & $\mathrm{i}$ & ind \\
\hline 195 & $\begin{array}{l}\text { Neidium iridis (Ehrb.) Cl. } \\
\text { var. iridis }\end{array}$ & NEIRIR & 1 & 0 & B & - & st & es & $\mathrm{o}-\mathrm{x}$ & $\mathrm{hb}$ & ind \\
\hline 196 & $\begin{array}{l}\text { Nitzschia acicularis } \\
\text { (Kütz.) W. Sm. }\end{array}$ & NI042A & 0 & 1 & P-B & temp & - & es & $\mathrm{o}-\mathrm{b}$ & $\mathrm{i}$ & alf \\
\hline 197 & Nitzschia amphibia Grun. & NIA01Y & $1-3$ & 1 & P-B & temp & - & $\mathrm{sp}$ & o & $\mathrm{i}$ & alf \\
\hline 198 & Nitzschia aurariae Choln. & NITZAQ & 0 & $1-6$ & B & - & - & - & - & oh & - \\
\hline 199 & $\begin{array}{l}\text { Nitzschia communis } \\
\text { Rabenh. }\end{array}$ & NITCOR & 2 & 0 & P-B & - & $\begin{array}{l}\text { st- } \\
\text { str }\end{array}$ & $\mathrm{sp}$ & o & $\mathrm{i}$ & alf \\
\hline 200 & $\begin{array}{l}\text { Nitzschia compressa var. } \\
\text { balatonis (Grun.) Lange- } \\
\text { Bert. }\end{array}$ & $\mathrm{NIC02Y}$ & 0 & $1-2$ & B & - & - & - & - & $\mathrm{hl}$ & \\
\hline 201 & $\begin{array}{l}\text { Nitzschia desertorum } \\
\text { Hust. }\end{array}$ & NITDSQ & 0 & $1-2$ & B & & $\begin{array}{l}\text { st- } \\
\text { str }\end{array}$ & - & $\mathrm{b}$ & $\mathrm{mh}$ & alf \\
\hline 202 & $\begin{array}{l}\text { Nitzschia frustulum var. } \\
\text { bulnheimiana (Rabenh.) } \\
\text { Grun. in Van Heurck }\end{array}$ & NI008A & $1-5$ & 1 & B & temp & - & $\mathrm{sp}$ & $\mathrm{b}$ & hl & alf \\
\hline
\end{tabular}




\begin{tabular}{|c|c|c|c|c|c|c|c|c|c|c|c|}
\hline No & Taxon & Code & Rud & Qish & Hab & $\mathbf{T}$ & Oxy & D & $\mathbf{S}$ & Hal & pH \\
\hline 203 & $\begin{array}{l}\text { Nitzschia grandifera } \\
\text { Hust. }\end{array}$ & NITGRO & 0 & 1 & $\mathrm{~B}$ & - & - & - & & $\mathrm{hl}$ & - \\
\hline 204 & $\begin{array}{l}\text { Nitzschia inconspicua } \\
\text { Grun. }\end{array}$ & NITINO & 0 & $1-5$ & B & - & - & es & $a-b$ & - & - \\
\hline 205 & Nitzschia laevis Hust. & NITLEO & 0 & 1 & B & - & - & - & - & $\mathrm{mh}$ & - \\
\hline 206 & $\begin{array}{l}\text { Nitzschia linearis (Ag.) } \\
\text { W. Sm. }\end{array}$ & NI031A & $1-4$ & 2 & B & temp & - & es & $\mathrm{x}$ & $\mathrm{i}$ & alf \\
\hline 207 & $\begin{array}{l}\text { Nitzschia monachorum } \\
\text { Lange-Bert. }\end{array}$ & NITMOQ & 0 & 1 & B & - & st & - & - & - & - \\
\hline 208 & Nitzschia obtusa W. Sm. & NI036A & 0 & $1-6$ & B & - & - & es & $\mathrm{b}$ & $\mathrm{mh}$ & - \\
\hline 209 & $\begin{array}{l}\text { Nitzschia palea (Kütz.) } \\
\text { W. Smit }\end{array}$ & NI017A & $1-6$ & $1-6$ & P-B & temp & - & $\mathrm{sp}$ & $0-x$ & $\mathrm{i}$ & ind \\
\hline 210 & $\begin{array}{l}\text { Nitzschia pseudofonticola } \\
\text { Hust. }\end{array}$ & NI028A & 1 & 1 & B & - & - & es & o-p & $\mathrm{i}$ & alf \\
\hline 211 & Nitzschia reversa $\mathrm{W}$. Sm. & NIR01Y & 0 & $1-3$ & $\mathrm{P}$ & - & - & - & - & $\mathrm{hl}$ & - \\
\hline 212 & $\begin{array}{l}\text { Nitzschia scalpelliformis } \\
\text { (Grun.) Grun. in Cleve et } \\
\text { Grun. }\end{array}$ & NISC1Y & 0 & $1-4$ & B & - & - & $\mathrm{sp}$ & - & $\mathrm{hl}$ & - \\
\hline 213 & $\begin{array}{l}\text { Nitzschia sigma (Kütz.) } \\
\text { W. Sm. }\end{array}$ & NIS01Y & 0 & $1-4$ & B & temp & - & es & - & $\mathrm{mh}$ & ind \\
\hline 214 & Nitzschia solita Hust. & NISL1Y & 0 & $1-3$ & B & - & st & es & $a-b$ & $\mathrm{mh}$ & alf \\
\hline 215 & $\begin{array}{l}\text { Nitzschia umbonata } \\
\text { (Ehrb.) Lange-Bert. }\end{array}$ & NI184A & 2 & 0 & $\mathrm{P}$ & - & $\begin{array}{l}\text { st- } \\
\text { str }\end{array}$ & es & b-o & $\mathrm{i}$ & ind \\
\hline 216 & $\begin{array}{l}\text { Nitzschia vermicularis } \\
\text { (Kütz.) Hantzsch in } \\
\text { Rabenh. }\end{array}$ & NI049A & 1 & 0 & B & - & - & - & o & $\mathrm{i}$ & alf \\
\hline 217 & Pinnularia borealis Ehrb. & PINBOQ & 2 & 1 & B & - & ae & es & $o-b$ & $\mathrm{i}$ & ind \\
\hline 218 & $\begin{array}{l}\text { Pinnularia brevicostata } \\
\mathrm{Cl} .\end{array}$ & PINBRR & 1 & 0 & B & cool & - & - & - & $\mathrm{i}$ & ind \\
\hline 219 & $\begin{array}{l}\text { Pinnularia gibba var. } \\
\text { linearis Hust. }\end{array}$ & PINGIR & 1 & 0 & B & - & - & - & - & $\mathrm{i}$ & ind \\
\hline 220 & $\begin{array}{l}\text { Pinnularia globiceps } \\
\text { Greg. }\end{array}$ & PINGLR & 1 & 0 & B & - & - & - & - & $\mathrm{i}$ & acf \\
\hline 221 & $\begin{array}{l}\text { Pinnularia infirma } \\
\text { Kramm. }\end{array}$ & PINPUR & 1 & 0 & B & - & - & - & - & $\mathrm{i}$ & ind \\
\hline 222 & $\begin{array}{l}\text { Pinnularia intermedia } \\
\text { (Lagerst.) Cleve }\end{array}$ & PI047A & 2 & 0 & B & - & st & - & $\mathrm{x}$ & $\mathrm{i}$ & ind \\
\hline 223 & $\begin{array}{l}\text { Pinnularia interrupta } \mathrm{W} . \\
\text { Smith }\end{array}$ & PINMER & $1-3$ & 0 & B & - & - & - & $0-\mathrm{x}$ & $\mathrm{i}$ & ind \\
\hline 224 & $\begin{array}{l}\text { Pinnularia major (Kütz.) } \\
\text { Rabenh. }\end{array}$ & PINMAR & $1-2$ & 0 & B & temp & $\begin{array}{l}\text { st- } \\
\text { str }\end{array}$ & & $\mathrm{x}$ & $\mathrm{i}$ & ind \\
\hline 225 & $\begin{array}{l}\text { Pinnularia microstauron } \\
\text { var. brebissonii (Ehrb.) } \\
\text { Cleve }\end{array}$ & PIMIBL & 2 & 0 & B & - & $\begin{array}{l}\text { st- } \\
\text { str }\end{array}$ & es & $\mathrm{o}-\mathrm{x}$ & $\mathrm{i}$ & ind \\
\hline 226 & $\begin{array}{l}\text { Pinnularia microstauron } \\
\text { (Ehrb.) Cleve var. } \\
\text { microstauron }\end{array}$ & PINMMR & $1-3$ & 0 & B & temp & - & $\mathrm{sp}$ & $\mathrm{x}$ & $\mathrm{i}$ & ind \\
\hline 227 & $\begin{array}{l}\text { Pinnularia viridis } \\
\text { (Nitzsch) Ehr. }\end{array}$ & PINVIR & $1-3$ & 0 & P-B & temp & - & - & $0-\mathrm{x}$ & $\mathrm{i}$ & ind \\
\hline 228 & $\begin{array}{l}\text { Placoneis elginensis } \\
\text { (Greg.) E. Cox }\end{array}$ & PLACER & 2 & 0 & B & - & - & sx & $\mathrm{x}-\mathrm{O}$ & $\mathrm{i}$ & ind \\
\hline 229 & $\begin{array}{l}\text { Placoneis gastrum } \\
\text { (Ehrb.) Mereschk. }\end{array}$ & PLAGAR & 5 & 0 & B & - & - & sx & $\mathrm{x}-\mathrm{O}$ & $\mathrm{i}$ & ind \\
\hline 230 & $\begin{array}{l}\text { Placoneis placentula } \mathrm{f} . \\
\text { rostrata A. Mayer }\end{array}$ & PLAPLR & 1 & 0 & B & - & - & - & - & $\mathrm{i}$ & alf \\
\hline
\end{tabular}




\begin{tabular}{|c|c|c|c|c|c|c|c|c|c|c|c|}
\hline No & $\begin{array}{c}\text { Taxon } \\
\end{array}$ & Code & Rud & Qish & Hab & $\mathbf{T}$ & Oxy & D & $\mathbf{S}$ & Hal & pH \\
\hline 231 & $\begin{array}{l}\text { Pleurosigma salinarum } \\
\text { Grun. }\end{array}$ & PL050A & 0 & $1-6$ & $\mathrm{~B}$ & - & - & - & - & $\mathrm{mh}$ & \\
\hline 232 & $\begin{array}{l}\text { Pleurosira laevis (Ehrb.) } \\
\text { Compère }\end{array}$ & PLELEQ & 0 & $1-6$ & B & temp & - & - & - & $\mathrm{mh}$ & alf \\
\hline 233 & $\begin{array}{l}\text { Reimeria sinuata (Greg.) } \\
\text { Kociolek et Stoermer }\end{array}$ & REIMER & $1-3$ & 0 & $\mathrm{~B}$ & - & st & $\mathrm{sx}$ & - & i & ind \\
\hline 234 & $\begin{array}{l}\text { Rhoicosphenia } \\
\text { abbreviata (C. Ag.) } \\
\text { Lange-Bert. }\end{array}$ & $\mathrm{RC} 002 \mathrm{~A}$ & 0 & $1-2$ & P-B & - & - & es & $\mathrm{x}-\mathrm{O}$ & $\mathrm{i}$ & alf \\
\hline 235 & $\begin{array}{l}\text { Rhopalodia brebissonii } \\
\text { Kramm. }\end{array}$ & RHOPAQ & 0 & 1 & B & - & - & & & hl & \\
\hline 236 & $\begin{array}{l}\text { Rhopalodia gibba (Ehrb.) } \\
\text { O. Müll. }\end{array}$ & RHPG1Y & 1 & 0 & B & temp & - & es & $\mathrm{x}-\mathrm{O}$ & $\mathrm{i}$ & alb \\
\hline 237 & $\begin{array}{l}\text { Sellaphora bacillum } \\
\text { (Ehrb.) D.G. Mann }\end{array}$ & NAVBAR & $1-2$ & 0 & B & - & - & sx & $o-b$ & $\mathrm{i}$ & alf \\
\hline 238 & $\begin{array}{l}\text { Sellaphora pupula } \\
\text { (Kütz.) Mereschk. }\end{array}$ & SELLUL & $1-2$ & 0 & B & eterm & st & $\mathrm{sp}$ & $0-\mathrm{x}$ & hl & ind \\
\hline 239 & $\begin{array}{l}\text { Sellaphora rectangularis } \\
\text { (Greg.) Lange-Bert. et } \\
\text { Metzeltin }\end{array}$ & SELLRL & $1-2$ & 0 & B & temp & - & sx & - & hl & ind \\
\hline 240 & $\begin{array}{l}\text { Stauroneis anceps Ehrb. } \\
\text { var. anceps }\end{array}$ & STAUAR & $2-3$ & 0 & P-B & - & - & sx & $\mathrm{x}$ & $\mathrm{i}$ & ind \\
\hline 241 & $\begin{array}{l}\text { Stauroneis anceps var. } \\
\text { linearis Rabenh. }\end{array}$ & STAALR & 1 & 0 & B & - & - & sx & $\mathrm{b}$ & $\mathrm{i}$ & alf \\
\hline 242 & $\begin{array}{l}\text { Stauroneis } \\
\text { phoenicenteron (Nitzsch) } \\
\text { Ehrb. }\end{array}$ & STAPHR & 1 & 0 & B & temp & - & es & $\mathrm{x}-\mathrm{O}$ & $\mathrm{i}$ & ind \\
\hline 243 & $\begin{array}{l}\text { Stauroneis producta } \\
\text { Grun. }\end{array}$ & STAUPQ & 0 & 1 & B & - & st & sx & o & $\mathrm{mh}$ & ind \\
\hline 244 & Stauroneis smithii Grun. & SA003A & 1 & 0 & P-B & - & $\begin{array}{l}\text { st- } \\
\text { str }\end{array}$ & & $\mathrm{x}-\mathrm{O}$ & $\mathrm{i}$ & alf \\
\hline 245 & $\begin{array}{l}\text { Staurosirella } \\
\text { leptostauron (Ehrb.) } \\
\text { Williams et Round }\end{array}$ & STRSLL & $3-5$ & 0 & B & - & st & es & $a-b$ & $\mathrm{hb}$ & alf \\
\hline 246 & $\begin{array}{l}\text { Staurosirella pinnata } \\
\text { (Ehrb.) Williams et } \\
\text { Round }\end{array}$ & STRSPL & $2-3$ & 0 & B & temp & $\begin{array}{l}\text { st- } \\
\text { str }\end{array}$ & es & b-a & $\mathrm{hl}$ & alf \\
\hline 247 & $\begin{array}{l}\text { Stephanodiscus } \\
\text { hantzschii Grun. in Cl. et } \\
\text { Grun. }\end{array}$ & STH01Y & 0 & $1-4$ & $\mathrm{P}$ & temp & st & es & $a-b$ & $\mathrm{i}$ & alf \\
\hline 248 & Surirella angusta Kütz. & SU001A & $1-3$ & 1 & P-B & - & $\begin{array}{l}\text { st- } \\
\text { str }\end{array}$ & es & o & $\mathrm{i}$ & alf \\
\hline 249 & $\begin{array}{l}\text { Surirella biseriata Bréb. } \\
\text { ex Godey }\end{array}$ & SURBIR & 1 & 0 & P-B & - & $\begin{array}{l}\text { st- } \\
\text { str }\end{array}$ & sx & $\mathrm{o}-\mathrm{b}$ & $\mathrm{i}$ & alf \\
\hline 250 & $\begin{array}{l}\text { Surirella brebissonii } \\
\text { Kramm. et Lange-Bert. }\end{array}$ & SUBREQ & 1 & 1 & B & - & $\begin{array}{l}\text { st- } \\
\text { str }\end{array}$ & - & $\mathrm{x}$ & $\mathrm{i}$ & alf \\
\hline 251 & $\begin{array}{l}\text { Surirella capronii Bréb. } \\
\text { ex Kütz. }\end{array}$ & SURCAO & 0 & 1 & P-B & - & st & & $\mathrm{x}$ & $\mathrm{i}$ & ind \\
\hline 252 & $\begin{array}{l}\text { Surirella minuta Bréb. in } \\
\text { Kütz. }\end{array}$ & SURMIR & $1-4$ & 0 & B & - & $\begin{array}{l}\text { st- } \\
\text { str }\end{array}$ & es & o-a & $\mathrm{i}$ & ind \\
\hline 253 & Surirella ovalis Bréb. & SUV01Y & 0 & $1-3$ & P-B & - & $\begin{array}{l}\text { st- } \\
\text { str }\end{array}$ & es & $\mathrm{o}$ & $\mathrm{mh}$ & alf \\
\hline 254 & Surirella peisonis Pant. & SUPIEQ & 0 & 1 & B & - & - & - & $\mathrm{a}$ & - & - \\
\hline 255 & $\begin{array}{l}\text { Surirella splendida } \\
\text { (Ehrb.) Kütz. }\end{array}$ & SURSPR & $1-2$ & 0 & P-B & - & - & - & $o-b$ & $\mathrm{i}$ & alf \\
\hline 256 & Surirella tenera Greg. & SURTER & 1 & 0 & P-B & - & st & es & o & $\mathrm{i}$ & alf \\
\hline
\end{tabular}




\begin{tabular}{|c|c|c|c|c|c|c|c|c|c|c|c|}
\hline No & Taxon & Code & Rud & Qish & Hab & $\mathbf{T}$ & Oxy & D & $\mathbf{S}$ & Hal & pH \\
\hline 257 & $\begin{array}{l}\text { Synedra inaequalis } \\
\text { Kobayasi }\end{array}$ & SYINAL & 1 & 0 & - & - & - & sX & - & - & - \\
\hline 258 & $\begin{array}{l}\text { Synedra ulna var. } \\
\text { amphirhynchus (Ehrb.) } \\
\text { Grun. }\end{array}$ & SYNUAR & $2-4$ & 0 & B & - & - & es & - & $\mathrm{i}$ & alf \\
\hline 259 & $\begin{array}{l}\text { Synedra ulna var. } \\
\text { contracta Østr. }\end{array}$ & SYNUCR & 1 & 0 & - & - & - & es & - & - & - \\
\hline 260 & $\begin{array}{l}\text { Tabellaria fenestrata } \\
\text { (Roth) Kütz. }\end{array}$ & TAFERR & $1-2$ & 0 & P-B & - & $\begin{array}{l}\text { st- } \\
\text { str }\end{array}$ & es & $\mathrm{x}$ & $\mathrm{hb}$ & acf \\
\hline 261 & $\begin{array}{l}\text { Tabellaria flocculosa } \\
\text { (Roth) Kütz. }\end{array}$ & TA001A & $1-2$ & 0 & P-B & eterm & $\begin{array}{l}\text { st- } \\
\text { str }\end{array}$ & es & $\mathrm{o}-\mathrm{a}$ & $\mathrm{hb}$ & acf \\
\hline 262 & $\begin{array}{l}\text { Thalassiosira weissflogii } \\
\text { (Grun. in Van Heurck) } \\
\text { Fryx. et Hasle }\end{array}$ & THAWEQ & 0 & $1-2$ & P-B & - & - & $\mathrm{sp}$ & o & hl & alf \\
\hline 263 & $\begin{array}{l}\text { Tryblionella acuminata } \\
\text { W. Sm. }\end{array}$ & TRYACA & 0 & $1-2$ & $\mathrm{P}$ & - & st & sx & b-p & $\mathrm{mh}$ & alf \\
\hline 264 & $\begin{array}{l}\text { Tryblionella apiculata } \\
\text { Greg. }\end{array}$ & TYA01Y & 0 & $1-6$ & B & - & - & es & o-a & $\mathrm{mh}$ & alf \\
\hline 265 & $\begin{array}{l}\text { Tryblionella debilis } \\
\text { Arnott in O'Meara }\end{array}$ & TRYBDQ & 0 & $1-3$ & P-B & - & ae & es & o & $\mathrm{i}$ & alf \\
\hline 266 & $\begin{array}{l}\text { Tryblionella gracilis } \mathrm{W} \text {. } \\
\text { Sm. }\end{array}$ & TYG01Y & 1 & $1-3$ & B & - & - & & $a-b$ & $\mathrm{hl}$ & alf \\
\hline 267 & $\begin{array}{l}\text { Tryblionella hungarica } \\
\text { (Grun.) Mann }\end{array}$ & TYH01Y & 0 & $1-6$ & P-B & - & - & $\mathrm{sp}$ & $a-b$ & $\mathrm{mh}$ & alf \\
\hline 268 & $\begin{array}{l}\text { Tryblionella punctata } \mathrm{W} \text {. } \\
\text { Sm. }\end{array}$ & TRYBPQ & 0 & 1 & B & eterm & - & - & - & $\mathrm{mh}$ & - \\
\hline 269 & $\begin{array}{l}\text { Ulnaria acus (Kütz.) } \\
\text { Aboal }\end{array}$ & FRAUAR & 1 & 0 & P-B & - & $\begin{array}{l}\text { st- } \\
\text { str }\end{array}$ & es & o-a & $\mathrm{i}$ & alb \\
\hline 270 & $\begin{array}{c}\text { Ulnaria ulna (Nitzsch) } \\
\text { Compère in Jahn et al. } \\
\text { Chlorophyta }\end{array}$ & FRU01Y & $1-5$ & $1-5$ & P-B & temp & $\begin{array}{l}\text { st- } \\
\text { str }\end{array}$ & es & b-o & $\mathrm{i}$ & ind \\
\hline 271 & $\begin{array}{l}\text { Actinastrum hantzschii } \\
\text { var. subtile Wolosz. }\end{array}$ & ACHS1Y & 0 & 1 & P-B & - & - & - & $\mathrm{b}$ & $\mathrm{i}$ & - \\
\hline 272 & $\begin{array}{l}\text { Characium } \\
\text { ornithocephalum A. Br. }\end{array}$ & COR01Y & 0 & 3 & Ep & - & - & - & - & $\mathrm{i}$ & - \\
\hline 273 & Chlamydomonas sp. & CHM01Y & 0 & 1 & $\mathrm{P}$ & - & - & - & $b-p$ & - & - \\
\hline 274 & Chlorococcum sp. & CHC01Y & 0 & $1-2$ & - & - & - & - & - & - & - \\
\hline 275 & $\begin{array}{l}\text { Cladophora glomerata } \\
\text { (L.) Kütz. }\end{array}$ & CLAG1Y & 0 & $1-6$ & P-B & - & $\begin{array}{l}\text { st- } \\
\text { str }\end{array}$ & - & b-o & $\mathrm{i}$ & alf \\
\hline 276 & $\begin{array}{l}\text { Closterium acerosum } \\
\text { (Schrank) Ehrb. ex Ralfs }\end{array}$ & CLSA1Y & 1 & 0 & P-B & - & $\begin{array}{l}\text { st- } \\
\text { str }\end{array}$ & - & $a-b$ & $\mathrm{i}$ & ind \\
\hline 277 & $\begin{array}{l}\text { Closterium peracerosum } \\
\text { Gay }\end{array}$ & CLOPEH & 0 & $1-4$ & P-B & - & $\begin{array}{l}\text { st- } \\
\text { str }\end{array}$ & - & $\mathrm{b}$ & - & - \\
\hline 278 & $\begin{array}{l}\text { Coelastrum astroideum } \\
\text { de Not. }\end{array}$ & COEA1Y & 0 & $1-5$ & $\mathrm{P}$ & - & $\begin{array}{l}\text { st- } \\
\text { str }\end{array}$ & - & $\mathrm{b}$ & - & - \\
\hline 279 & $\begin{array}{l}\text { Coelastrum microporum } \\
\text { Näg. }\end{array}$ & COEM1Y & 0 & 1 & P-B & - & $\begin{array}{l}\text { st- } \\
\text { str }\end{array}$ & - & $\mathrm{b}$ & $\mathrm{i}$ & ind \\
\hline 280 & $\begin{array}{l}\text { Cosmarium } \\
\text { subprotumidum Nordst. }\end{array}$ & COSSSQ & 0 & 1 & P-B & - & $\begin{array}{l}\text { st- } \\
\text { str }\end{array}$ & - & - & - & acf \\
\hline 281 & $\begin{array}{l}\text { Desmodesmus abundans } \\
\text { (Kirchn.) Hegew. }\end{array}$ & SCEQUR & $1-3$ & 0 & P-B & - & $\begin{array}{l}\text { st- } \\
\text { str }\end{array}$ & - & o-a & - & - \\
\hline 282 & $\begin{array}{l}\text { Desmodesmus armatus } \\
\text { var. armatus (Chod.) } \\
\text { Hegew. }\end{array}$ & DEA01Y & 0 & 1 & P-B & - & $\begin{array}{l}\text { st- } \\
\text { str }\end{array}$ & - & o-a & - & - \\
\hline
\end{tabular}




\begin{tabular}{|c|c|c|c|c|c|c|c|c|c|c|c|}
\hline No & $\begin{array}{c}\text { Taxon } \\
\end{array}$ & Code & Rud & Qish & Hab & $\mathbf{T}$ & Oxy & D & $\mathbf{S}$ & Hal & pH \\
\hline 283 & $\begin{array}{l}\text { Desmodesmus armatus } \\
\text { var. spinosus (Fritsch et } \\
\text { Rich) Hegew. }\end{array}$ & DESASH & 0 & 2 & $\mathrm{P}$ & - & $\begin{array}{l}\text { st- } \\
\text { str }\end{array}$ & - & $\mathrm{b}$ & - & - \\
\hline 284 & $\begin{array}{l}\text { Desmodesmus communis } \\
\text { (Hegew.) Hegew. }\end{array}$ & SCEQQL & $1-3$ & 0 & P-B & - & $\begin{array}{l}\text { st- } \\
\text { str }\end{array}$ & - & $\mathrm{b}$ & $\mathrm{i}$ & ind \\
\hline 285 & $\begin{array}{l}\text { Desmodesmus costato- } \\
\text { granulatus (Skuja) } \\
\text { Hegew. }\end{array}$ & DECG1Y & 0 & 2 & P-B & - & $\begin{array}{l}\text { st- } \\
\text { str }\end{array}$ & - & $\mathrm{b}$ & - & - \\
\hline 286 & $\begin{array}{l}\text { Desmodesmus } \\
\text { intermedius (R. Chod.) } \\
\text { Hegew. }\end{array}$ & DEIT1Y & 0 & 2 & P-B & - & $\begin{array}{l}\text { st- } \\
\text { str }\end{array}$ & - & $\mathrm{b}$ & - & - \\
\hline 287 & $\begin{array}{l}\text { Desmodesmus opoliensis } \\
\text { (P. Richt.) Hegew. }\end{array}$ & SCEOPR & 2 & 0 & P-B & - & $\begin{array}{l}\text { st- } \\
\text { str }\end{array}$ & - & $\mathrm{b}$ & - & - \\
\hline 288 & $\begin{array}{l}\text { Desmodesmus } \\
\text { protuberans (Fritsch et } \\
\text { Rich) Hegew. }\end{array}$ & DEP01Y & 0 & 1 & P-B & - & $\begin{array}{l}\text { st- } \\
\text { str }\end{array}$ & - & - & - & - \\
\hline 289 & $\begin{array}{l}\text { Desmodesmus spinosus } \\
\text { (R. Chod.) Hegew. }\end{array}$ & DES01Y & 0 & 1 & P-B & - & $\begin{array}{l}\text { st- } \\
\text { str }\end{array}$ & - & o-b & - & - \\
\hline 290 & Dunaliella sp. & DUNALQ & 0 & $1-6$ & - & - & - & - & - & - & - \\
\hline 291 & $\begin{array}{l}\text { Gloxidium rotatoriae } \\
\text { Korsch. }\end{array}$ & GLOXIRL & 1 & 0 & - & - & - & - & - & - & - \\
\hline 292 & Gongrosira sp. & GONGRQ & 0 & 1 & Ep & - & - & - & - & - & - \\
\hline 293 & $\begin{array}{l}\text { Microspora quadrata } \\
\text { Hazen }\end{array}$ & MICSPR & 5 & 0 & $\mathrm{~B}$ & - & - & - & $\mathrm{b}$ & - & - \\
\hline 294 & $\begin{array}{l}\text { Monoraphidium } \\
\text { contortum (Thur.) Kom.- } \\
\text { Legn. }\end{array}$ & MOCONA & 0 & 2 & P-B & - & $\begin{array}{l}\text { st- } \\
\text { str }\end{array}$ & - & $\mathrm{b}$ & - & - \\
\hline 295 & $\begin{array}{l}\text { Monoraphidium griffithii } \\
\text { (Berk.) Kom.-Legn. }\end{array}$ & MOG01Y & 1 & 1 & P-B & - & $\begin{array}{l}\text { st- } \\
\text { str }\end{array}$ & - & $\mathrm{b}$ & $\mathrm{i}$ & - \\
\hline 296 & $\begin{array}{l}\text { Monoraphidium } \\
\text { irregulare (G.M. Smith) } \\
\text { Komárkova-Legnerova }\end{array}$ & ANKANL & $1-2$ & 0 & $\mathrm{P}$ & - & - & - & - & $\mathrm{i}$ & - \\
\hline 297 & $\begin{array}{l}\text { Monoraphidium minutum } \\
\text { (Näg.) Kom.-Legn. }\end{array}$ & MOM01Y & 0 & 6 & P-B & - & $\begin{array}{l}\text { st- } \\
\text { str }\end{array}$ & - & b-a & - & - \\
\hline 298 & Mougeotia sp. ster. & MU001Y & $1-3$ & 0 & B & - & - & - & o & - & - \\
\hline 299 & Oedogonium sp. ster. & OE001Y & 1 & $2-4$ & B & - & - & - & - & - & - \\
\hline 300 & Oocystis lacustris Chod. & OOLA0A & 0 & 2 & P-B & - & $\begin{array}{l}\text { st- } \\
\text { str }\end{array}$ & - & b-o & $\mathrm{hl}$ & - \\
\hline 301 & $\begin{array}{l}\text { Oocystis submarina } \\
\text { Lagerh. }\end{array}$ & OOC01Y & 0 & 1 & P-B & - & st & - & - & $\mathrm{i}$ & - \\
\hline 302 & $\begin{array}{l}\text { Pandorina morum } \\
\text { (Müll.) Bory }\end{array}$ & PAM01Y & 0 & 4 & $\mathrm{P}$ & - & st & - & $\mathrm{b}$ & $\mathrm{i}$ & - \\
\hline 303 & $\begin{array}{l}\text { Pediastrum duplex } \\
\text { Meyen }\end{array}$ & PDD01Y & 0 & $3-4$ & $\mathrm{P}$ & - & $\begin{array}{l}\text { st- } \\
\text { str }\end{array}$ & - & $\mathrm{o}-\mathrm{a}$ & $\mathrm{i}$ & ind \\
\hline 304 & $\begin{array}{l}\text { Pediastrum tetras (Ehrb.) } \\
\text { Ralfs }\end{array}$ & PETETH & 0 & 2 & P-B & - & $\begin{array}{l}\text { st- } \\
\text { str }\end{array}$ & - & $\mathrm{o}-\mathrm{a}$ & $\mathrm{i}$ & ind \\
\hline 305 & $\begin{array}{l}\text { Raphidocelis sigmoidea } \\
\text { Hind. }\end{array}$ & RCS01Y & 0 & $1-4$ & $\mathrm{P}$ & - & $\begin{array}{l}\text { st- } \\
\text { str }\end{array}$ & - & - & - & - \\
\hline 306 & Raphidocelis sp. & RAPHIQ & 0 & 1 & - & - & - & - & - & - & - \\
\hline 307 & $\begin{array}{l}\text { Rhizoclonium } \\
\text { hieroglyphicum (Ag.) } \\
\text { Kütz. }\end{array}$ & RHZ01Y & $1-6$ & 6 & B & - & $\begin{array}{l}\text { st- } \\
\text { str }\end{array}$ & - & o & $\mathrm{hl}$ & - \\
\hline 308 & $\begin{array}{l}\text { Scenedesmus acuminatus } \\
\text { var. acuminatus (Lagerh.) } \\
\text { Chod. }\end{array}$ & SA001Y & $2-4$ & 1 & P-B & - & $\begin{array}{l}\text { st- } \\
\text { str }\end{array}$ & - & $\mathrm{b}$ & $\mathrm{i}$ & ind \\
\hline
\end{tabular}




\begin{tabular}{|c|c|c|c|c|c|c|c|c|c|c|c|}
\hline No & Taxon & Code & Rud & Qish & Hab & $\mathbf{T}$ & Oxy & D & $\mathbf{S}$ & Hal & pH \\
\hline 309 & $\begin{array}{l}\text { Scenedesmus acuminatus } \\
\text { var. biseriatus Reinh. }\end{array}$ & SCEACR & 2 & 0 & $\mathrm{P}$ & - & - & - & - & $\mathrm{i}$ & - \\
\hline 310 & $\begin{array}{l}\text { Scenedesmus bijugatus } \\
\text { (Turp.) Kütz. }\end{array}$ & SCEBIR & 2 & 0 & $\mathrm{P}$ & - & - & - & $\mathrm{o}-\mathrm{a}$ & $\mathrm{i}$ & ind \\
\hline 311 & $\begin{array}{l}\text { Scenedesmus } \\
\text { incrassatulus Bohl. }\end{array}$ & SCEINH & 0 & $1-3$ & P-B & - & $\begin{array}{l}\text { st- } \\
\text { str }\end{array}$ & - & - & - & - \\
\hline 312 & $\begin{array}{l}\text { Scenedesmus obliquus } \\
\text { var. alternans } \text { Christjuk }\end{array}$ & SCEOAL & $1-4$ & 0 & - & - & - & - & - & - & - \\
\hline 313 & $\begin{array}{l}\text { Scenedesmus obliquus } \\
\text { var. obliquus (Turp.) } \\
\text { Kütz. }\end{array}$ & SCEOBQ & 1 & 1 & P-B & - & st & - & b-p & $\mathrm{i}$ & - \\
\hline 314 & $\begin{array}{l}\text { Scenedesmus obtusus } \\
\text { Meyen }\end{array}$ & SO001Y & 0 & 2 & P-B & - & $\begin{array}{l}\text { st- } \\
\text { str }\end{array}$ & - & b & - & - \\
\hline 315 & $\begin{array}{l}\text { Schroederia setigera } \\
\text { (Schröd.) Lemm. }\end{array}$ & SDS01Y & $1-2$ & 0 & $\mathrm{P}$ & - & $\begin{array}{l}\text { st- } \\
\text { str }\end{array}$ & - & $b-o$ & $\mathrm{i}$ & - \\
\hline 316 & Spirogyra sp. ster. & SPG01Y & $3-6$ & 4 & B & - & & - & - & - & - \\
\hline 317 & $\begin{array}{l}\text { Stigeoclonium tenue } \\
\text { (Ag.) Kütz. }\end{array}$ & SCT01Y & $1-6$ & 0 & B & - & $\begin{array}{l}\text { st- } \\
\text { str }\end{array}$ & - & b-p & - & - \\
\hline 318 & $\begin{array}{l}\text { Stylosphaeridium } \\
\text { epiphyticum (Korsch.) } \\
\text { Korsch. }\end{array}$ & STYE1Y & 0 & 2 & Ep & - & - & - & - & - & - \\
\hline 319 & $\begin{array}{l}\text { Tetraedron minimum (A. } \\
\text { Br.) Hansg. }\end{array}$ & TETMMA & 0 & $1-2$ & & - & - & - & - & - & - \\
\hline 320 & Ulothrix tenerrima Kütz. & ULOTTL & $2-6$ & 0 & B & - & - & - & $\mathrm{o}-\mathrm{a}$ & $\mathrm{i}$ & - \\
\hline 321 & $\begin{array}{l}\text { Ulothrix zonata (Weber } \\
\text { et Mohr) Kütz. }\end{array}$ & ULZONL & $1-6$ & 0 & P-B & - & $\begin{array}{l}\text { st- } \\
\text { str }\end{array}$ & - & $\mathrm{o}-\mathrm{a}$ & $\mathrm{i}$ & ind \\
\hline 322 & $\begin{array}{l}\text { Uronema confervicolum } \\
\text { Lagerh. } \\
\text { Rhodophyta }\end{array}$ & URC01Y & 0 & $2-4$ & B & - & $\begin{array}{l}\text { st- } \\
\text { str }\end{array}$ & - & $\mathrm{o}-\mathrm{a}$ & - & - \\
\hline 323 & $\begin{array}{l}\text { Audouinella pygmaea } \\
\text { (Kütz.) Weber-van Bosse }\end{array}$ & AUDOUO & 0 & $5-6$ & B & - & str & - & $\mathrm{x}-\mathrm{O}$ & - & alf \\
\hline
\end{tabular}

Note: Hab - habitat (Ep-epiphytes, $B$ - benthic, $P$ - B - plankto-benthic, $P$ - planktonic); $T$-temperature (eterm-eurytermic; temp-temperate; warm-warm water; cool-cool water); Oxy-streaming and oxygenation (st - standing water; st-str - low streaming water; str-streaming water); $S$-degree of saprobity on the Pantle-Buck's (Pantle \& Buck 1955) ( $x$-xenosaprobes, $x$-o -xeno-oligosaprobes, o- $x$ - oligoxenosaprobes, $x$ - $\beta$-xseno-betamesosaprobes, $o$-oligosaprobes, o- $\beta$-oligo-betamesosaprobes, $\beta$-o - betaoligosaprobes, o- $\alpha$-oligo-alphamesosaprobes, $\beta$ - betamesosaprobes, $\beta$ - $\alpha$ - beta-alphamesosaprobes, $\alpha-\beta$ alpha-betamesosaprobes, $\alpha$-alphamesosaprobes); $D$ - degree of saprobity on the Watanabe's (Watanabe 1986) (sx - saproxenous, es - eurysaprobes, sp - saprophiles); Hal - halobity degree (hb - oligohalobeshalophobes, $i$-oligohalobes-indifferent, $m h$ - mesohalobes, $h l$ - halophiles, $p h$ - polyhalobes); $\mathrm{pH}-\mathrm{pH}$ degree (alf-alkaliphiles, ind-indifferents; acf-acidophiles, alb-alcalibiontes). 


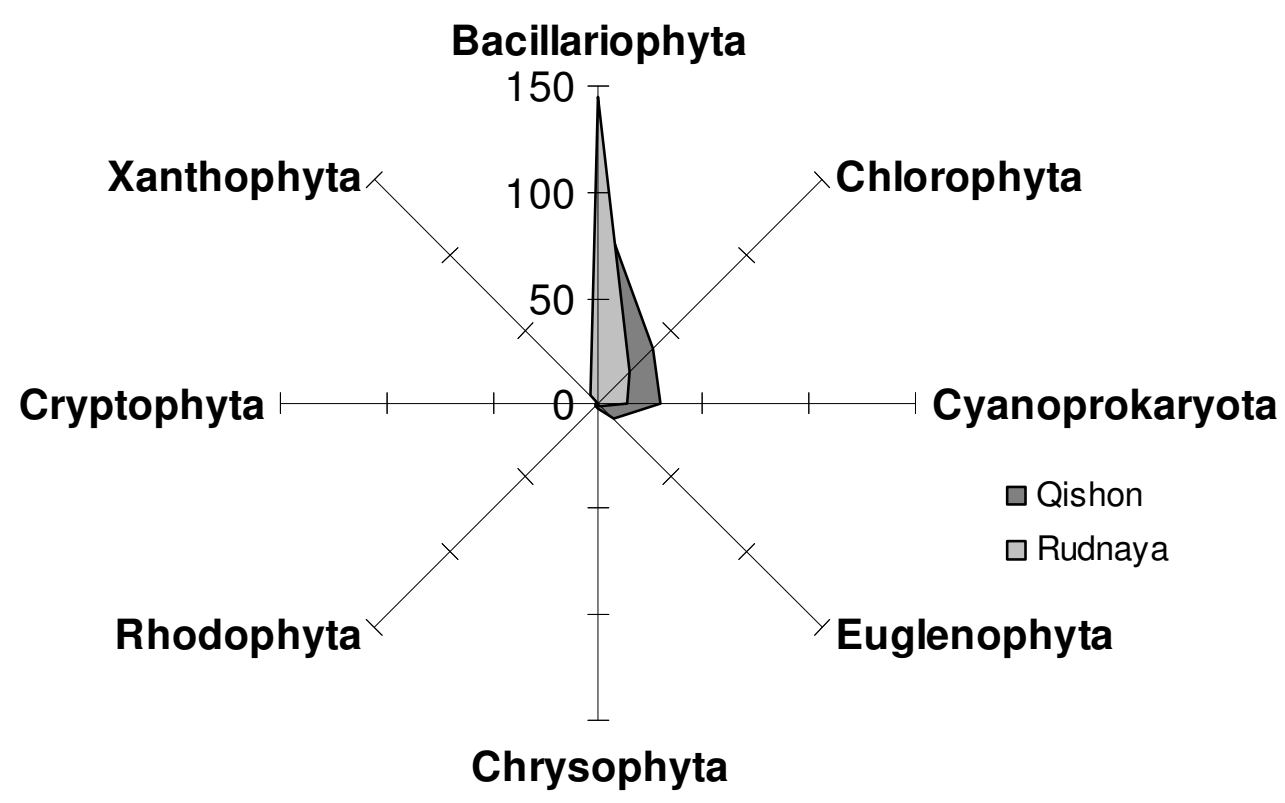

Figure 3. Distribution of the species community between the algal Divisions in the Rudnaya River and Qishon River.

The diversity of the species is similar in the two rivers with diatoms prevailing. Figure 3 shows that green algae are second in importance in the both rivers, followed by the cyanoprokaryotes. The presence of red algae can be noted as a unique element of the Qishon River algoflora.

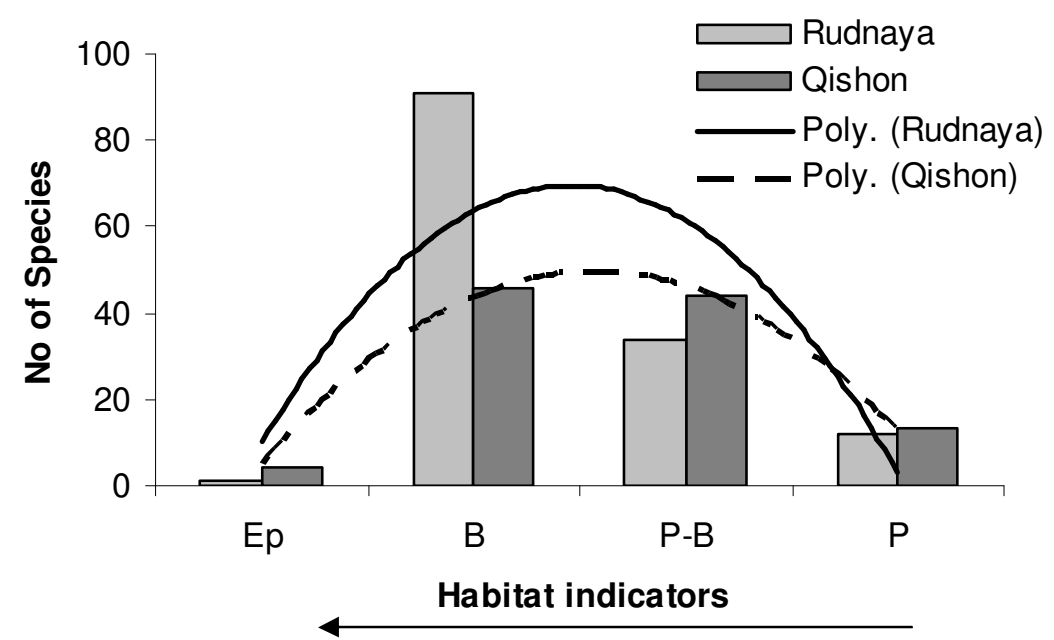

Figure 4. Distribution of the species diversity in both rivers between habitat ecological groups.

Distribution of species diversity over the substrate habitation groups in both rivers is shown in Figure 4. The arrow specifies a direction of strengthening of communication with substrate. In the Rudnaya River, the substrate bound species prevail. In the Qishon River, the benthic and plankto-benthic algae are represented by almost even species numbers because the river is low streaming. However the trend lines for the both distributions are similar. Their peaks occur between the groups of benthic and planktobenthic algae. 


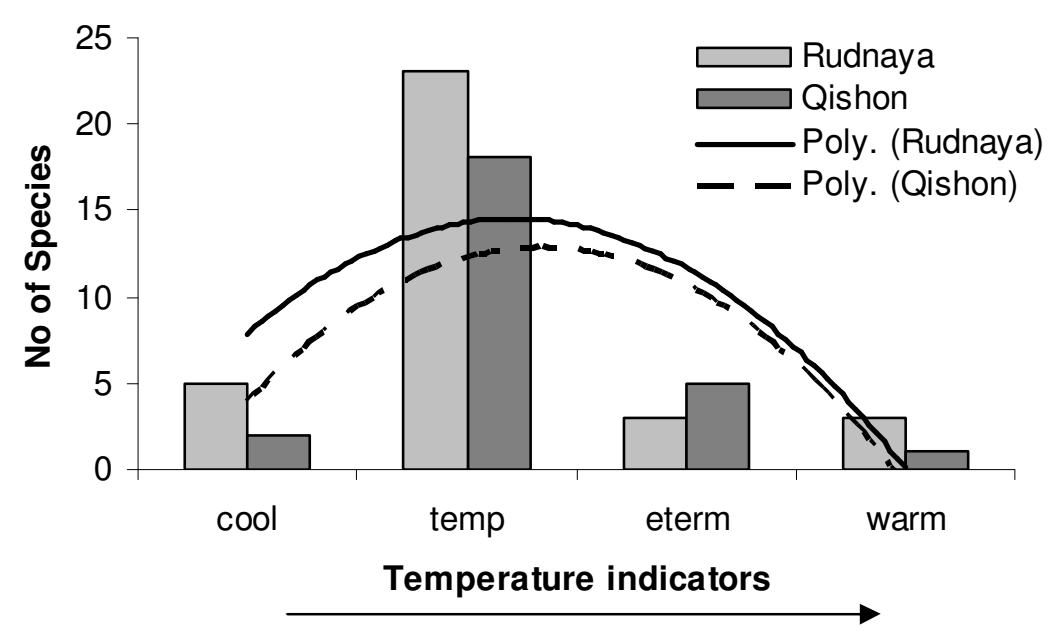

Figure 5. Distribution of the species diversity in both rivers between temperature indicator groups.

The whole range of the temperature indicators is represented (Figure 5), predominated by the temperate indicators in both rivers. The arrow specifies strengthening parameter. The trend lines of both distributions are similar, and their peaks occur between the groups of temperate and eurythermic algae.

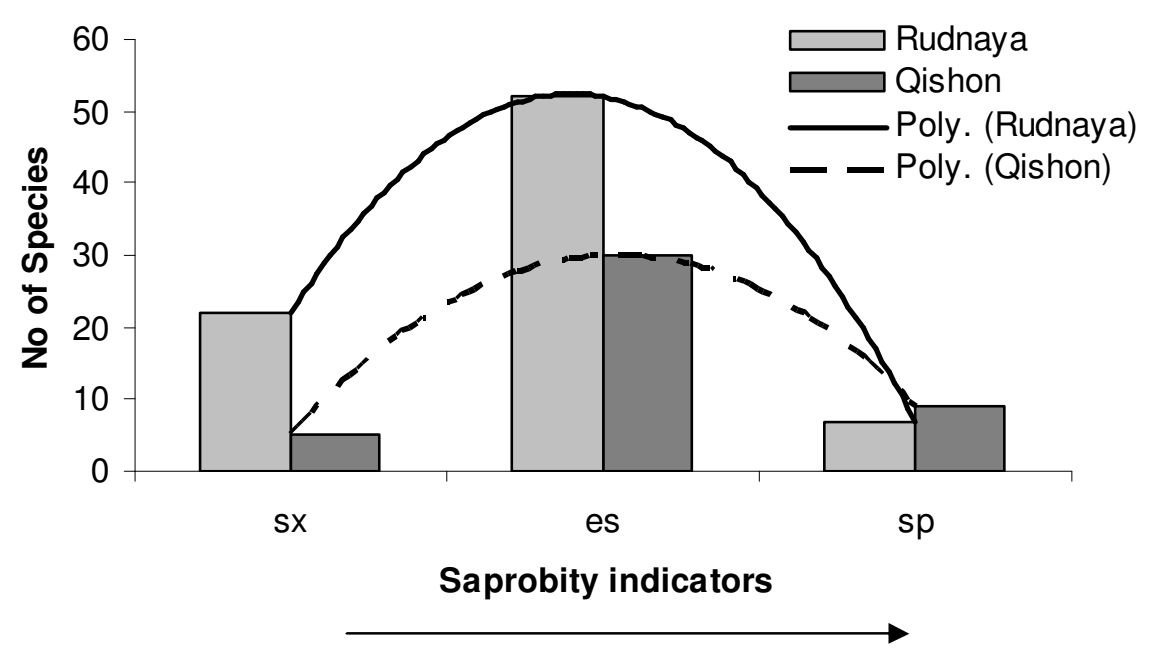

Figure 6. Distribution of the species diversity in both rivers between indicator groups of organic pollution (Watanabe's system).

Figure 6 shows distribution of saprobity indicators defined by Watanabe's method (1986). The arrow specifies strengthening parameter. Both distributions are dominated by eurysaprobic group; however the peak for the Qishon River is displaced toward the saprophile diversity, hence, these indicators show a somewhat greater organic pollution in the Qishon River, than in the Rudnaya River. 


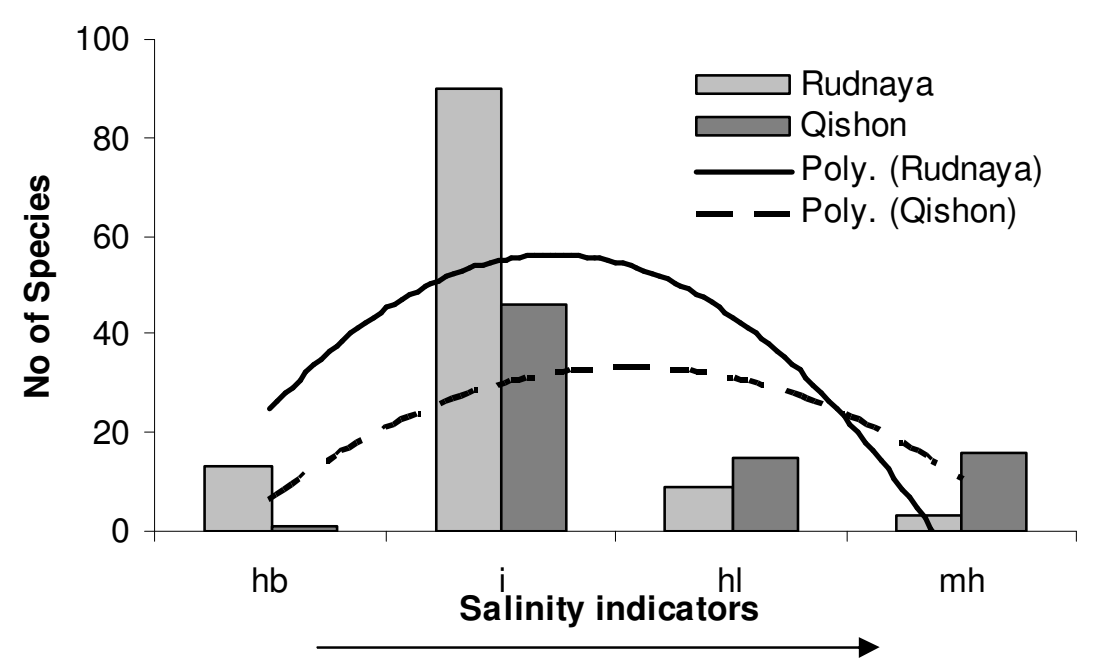

Figure 7. Distribution of the species diversity in both rivers between salinity indicator groups.

Salinity indicators represent the oligohalobe and medohalobe groups for both rivers (Figure 7). The arrow specifies strengthening parameter. Among the oligohalobes, the group of indifferents dominates in both rivers. The peak of a trend line for the Qishon River is somewhat displaced towards the indicators of high chloride concentrations. The group of mesohalobes in the Qishon River is also relatively prominent. It testifies to an appreciable influence of marine tidal waters on the algal diversity in the Qishon River, whereas the waters of the Rudnaya River are fresher.

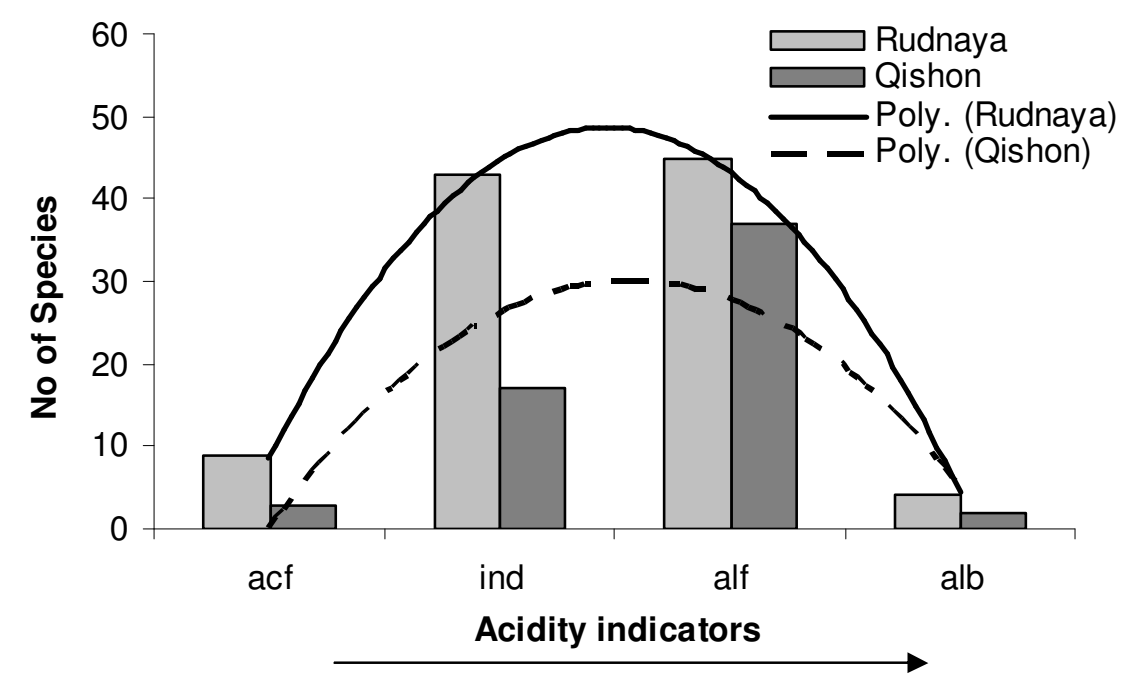

Figure 8. Distribution of the species diversity in both rivers between acidity indicator groups.

Algae in both rivers represent the whole spectrum of $\mathrm{pH}$ indicators (Figure 8). The arrow specifies strengthening parameter. In the Rudnaya River the group indifferents, preferring neutral waters, predominates. In the Qishon River a prevalence of alcalic species is better marked. Hence, with due attention to the peaks of the trend lines, we conclude that the algae from the Rudnaya River show preference of neutral, subacidic 
or alkalic waters characteristic of silicate bedrocks, whereas in the Qishon River indicators reflect the regional norm for carbonate provinces (Meybeck \& Helmer 1989).

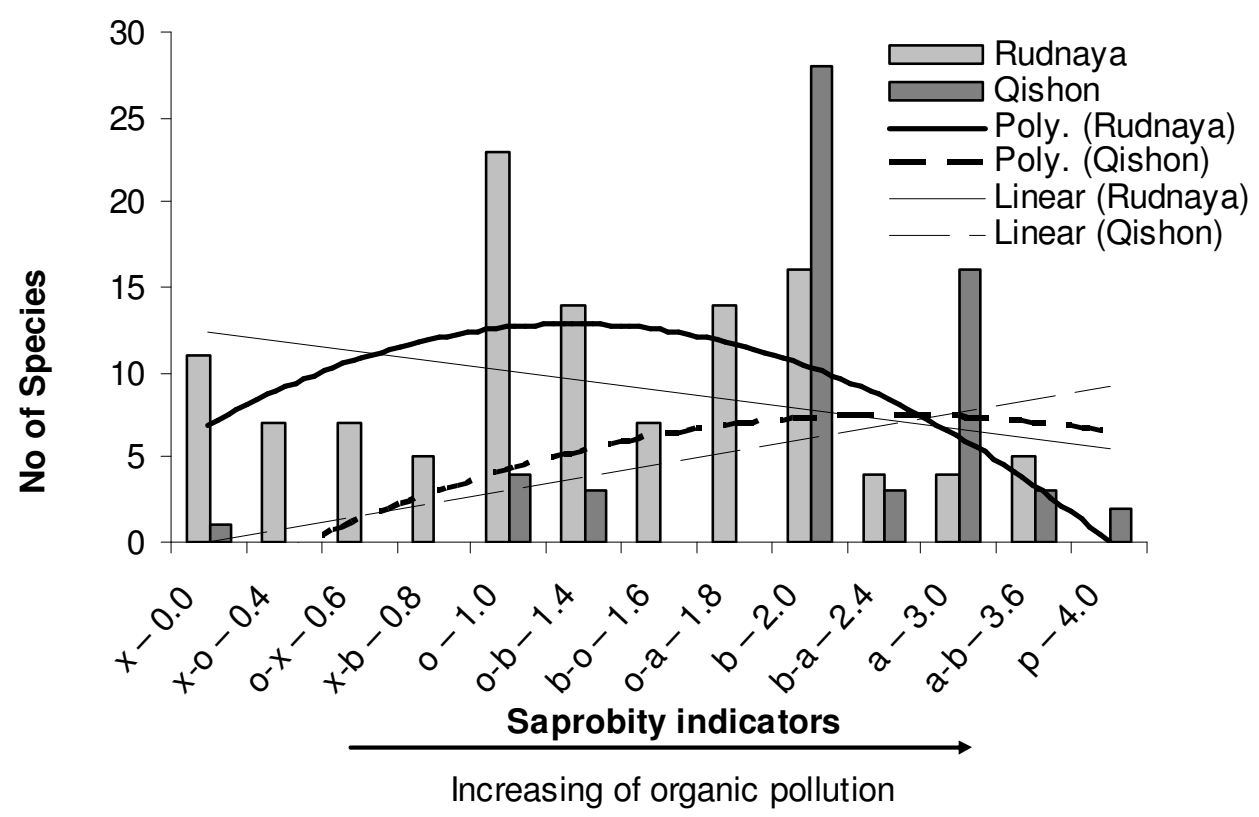

Figure 9. Distribution of the species diversity in both rivers between indicator groups of organic pollution (Sládeček system).

The indicators of organic pollution defined by the method of Pantle and Buck (1955) modified by Sládeček (1986) came from a wide range of ecological groups (Figure 9). The arrow specifies strengthening parameter.

In the Rudnaya River, the group of oligosaprobiontes is the largest, whereas in the Quishon River, the betamesosaprobiontes is better represented. The polynomial trend lines reflect a greater number of clear-water species in the Rudnaya River, while the indicators of moderate pollution are more numerous in the Quishon River. The oligoand xenosaprobic groups are particularly prominent in the Rudnaya River, while the alphmesosaprobic species are more prominent in the Qishon River, which is fairly obvious when the line trends are compared. Therefore, the organic content is greater in the Qishon then in the Rudnaya River.

At the same time, the polluted sections of these rivers differ in the composition of pollutants. The Rudnaya River is clear in the upper reaches being polluted down of Dal'negorsk industrial town. In distinction, the Qishon River is most heavily polluted by agricultural wastes in the upper reaches, with the industrial wastes added downstream. These distinctions are reflected by the dynamics of the saprobity indices in both rivers. 


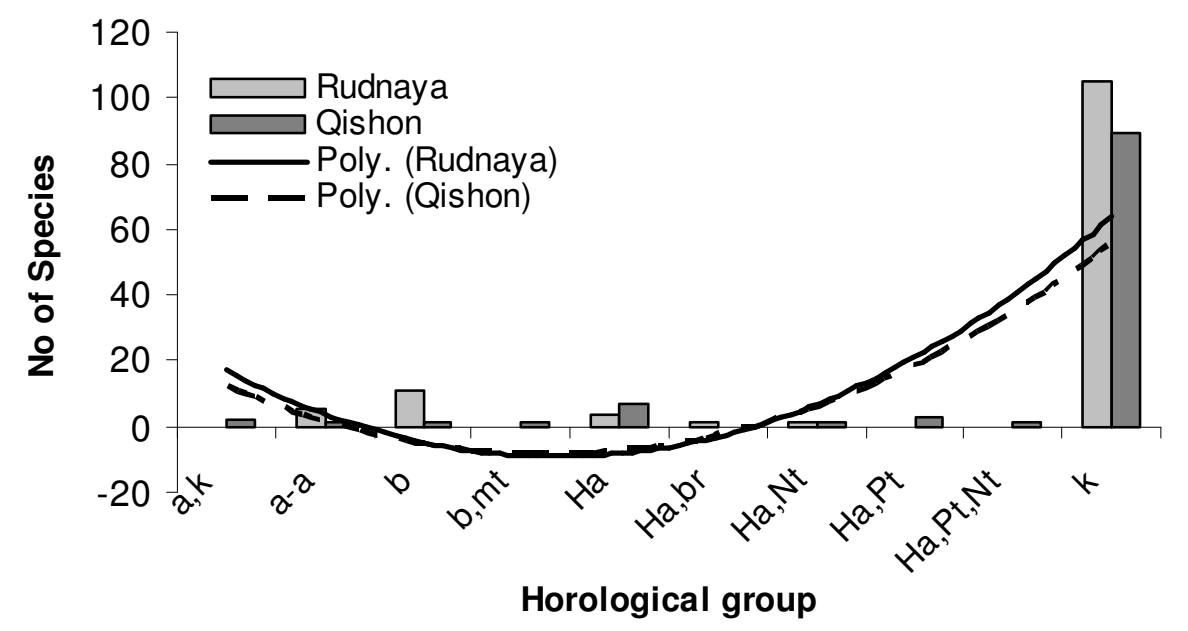

Figure 10. Distribution of the species diversity in both rivers between chorological groups.

For geographical analysis, we revealed differentiation of algal species over the major chorological groups (Figure 10). Despite the minor differences in the content of the boreal and holarctic species, the trend lines are similar for both river being dominated by cosmopolitan species.

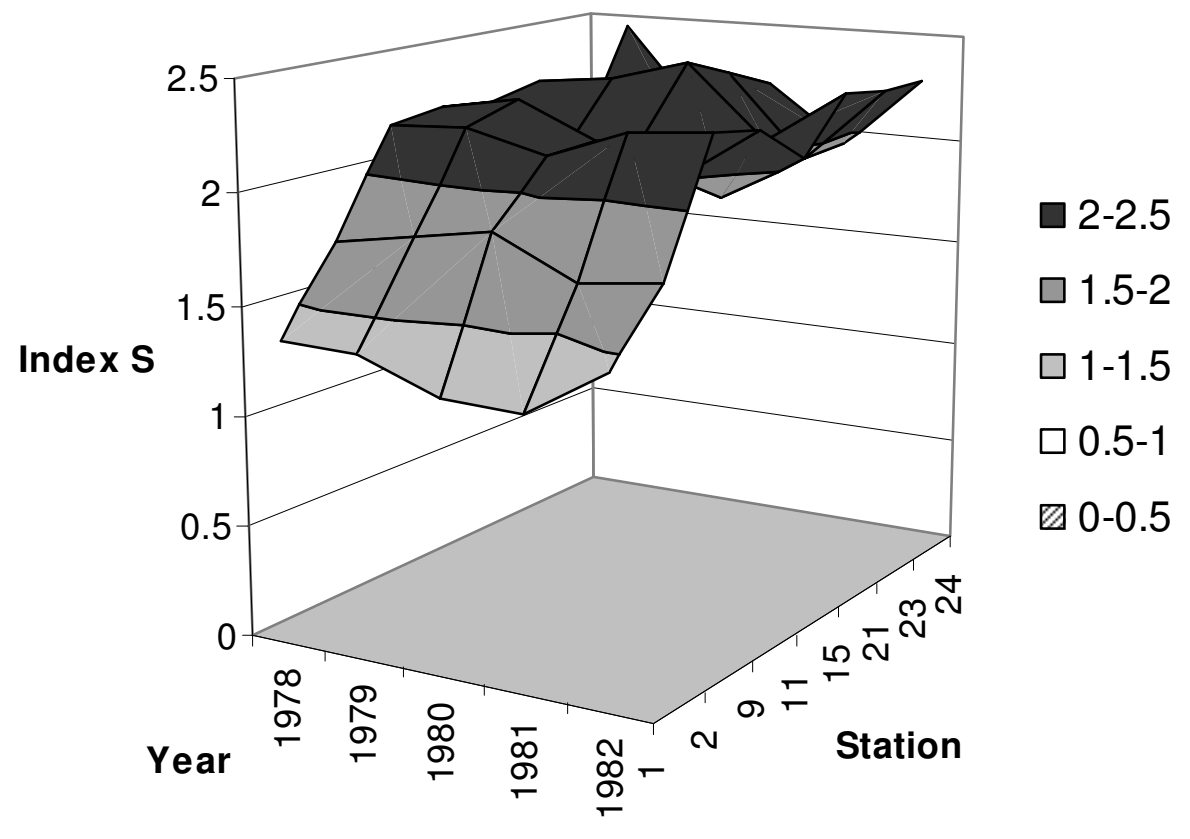

Figure 11. Saprobity index S dynamic over the sampling station of the Rudnaya River.

Fig. 11 shows that the saprobity index $\mathrm{S}$ for the Rudnaya River (Barinova, Medvedeva 1989;1992a,b; Medvedeva et al. 1986b) fluctuates from the oligosaprobic zone, Class II in the upper reaches (where indices ranged 0.5-1.5) to the betamesosaprobic zone, and Class III near the mouth (where indices ranged 1.5-2.5). During the whole period of observation, the concentration of organic pollutants tended 
to increase from the upper reaches towards the mouth, where we found a weak 2-year cyclicity of the index.

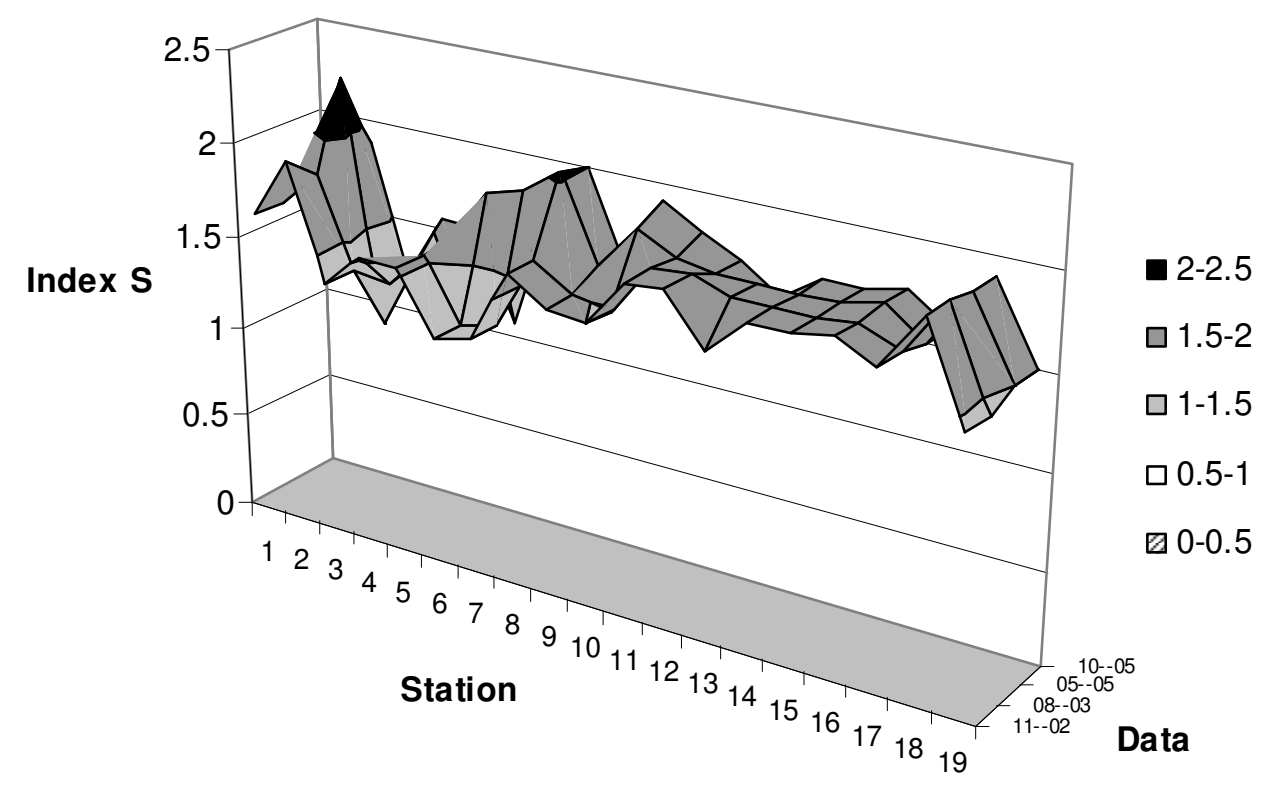

Figure 12. Saprobity index S dynamic over the sampling station of the Qishon River.

Fig 12 shows the dynamics of the saprobity index $\mathrm{S}$ in the Qishon River during the period 2002 - 2005 (Barinova et al. 2004). The index fluctuates, as in the Rudnaya River, from the oligosaprobic zone, Class II to the betamesosaprobic zone, Class III. The least polluted water was found in the upper reaches (where indices ranged 1.431.5). Then, across the agricultural area and before the industrial zone of Haifa the water quality degraded while fluctuations of the index increased (1.93-2.04). This is considered as evidence of misbalance of the system over this segment. At stations 2 and 3 , over the zone of industrial toxic wastes, the saprobity index decreased (1.0-1.36) then rising again toward its maximal values in the tidal zone (2.29).

The dynamics of the saprobiyty indices in both rivers show a lesser anthropogenic impact for Rudnaya River than for Qishon River. This is attested by the low amplitude fluctuations and the evidence of natural cyclicity in the Rudnaya River, whereas in the Qishon River the fluctuations are greater and the pollution is more dramatic in the upper reaches. 


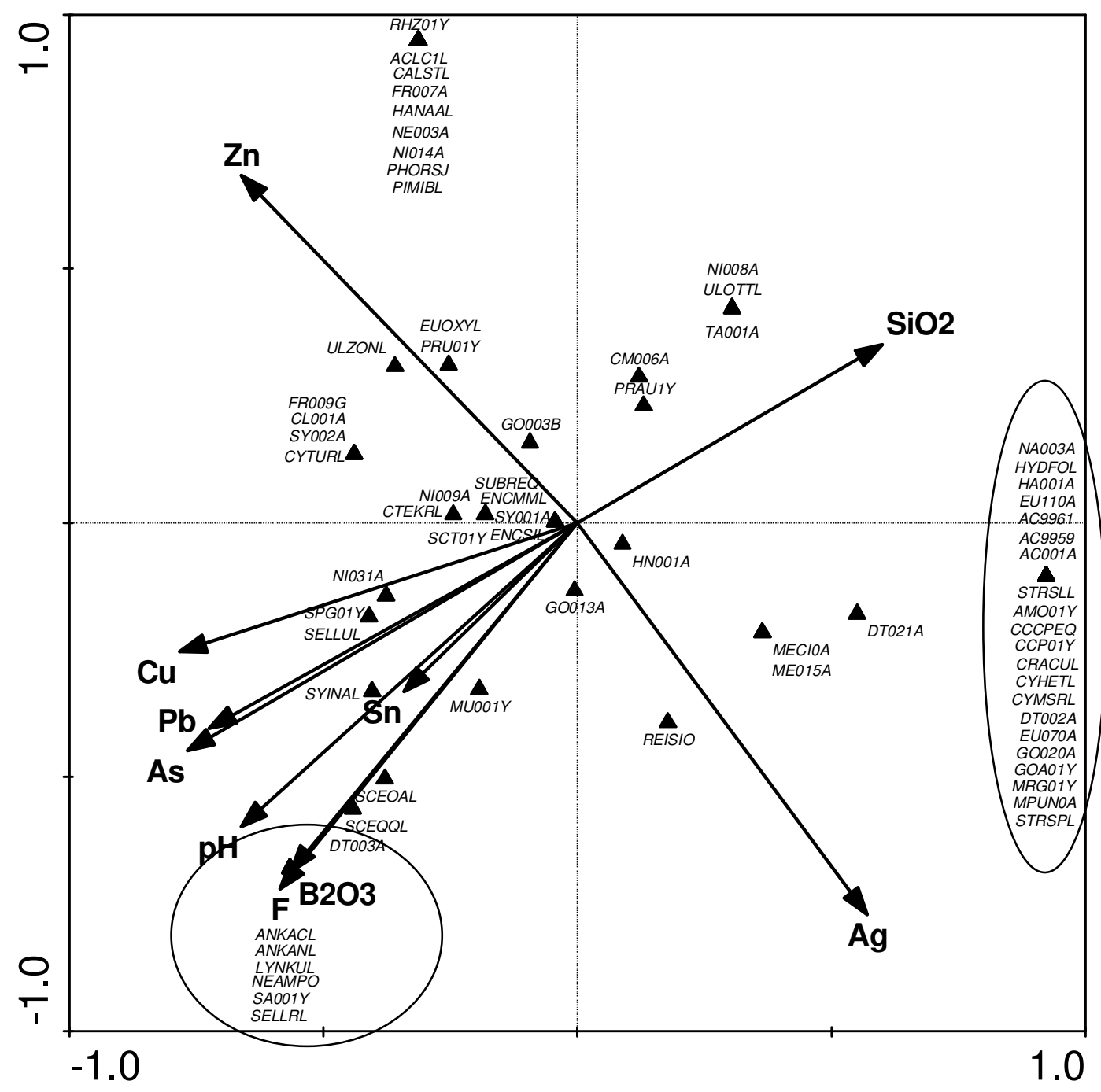

Figure 13. Biplot of environmental variables and species scores in a CCA of the Rudnaya River. Full names of species are presented in Table 3.

Figure 13 presents the main factors influencing algal species abundance in the Rudnaya River. It can be seen that the borate salts, fluorides, copper, lead and their associated arsenic all have the same anthropogenic source. It seems that their concentration is related to the $\mathrm{pH}$ fluctuations. In distinction, both zinc and silver are of natural origins and their influence on biodiversity is different, even opposite to that of anthropogenic elements.

As a whole, the species of polluted waters are fairly tolerant to individual pollutants, which is in sharp contrast to the group of the neutral $\mathrm{pH}$ clear-water species (marked out by the ellipse to the right).

Marked by the lower left ellipse is a biosensor group sensitive to borates and fluorides. This group includes the green algae Ankistrodesmus acicularis, A. angustus, Scenedesmus acuminatus, that are used for biotesting of toxicants, the blue-green algae Lyngbya kuetzingii and also the diatoms Neidium ampliatum and Sellaphora rectangularis. 


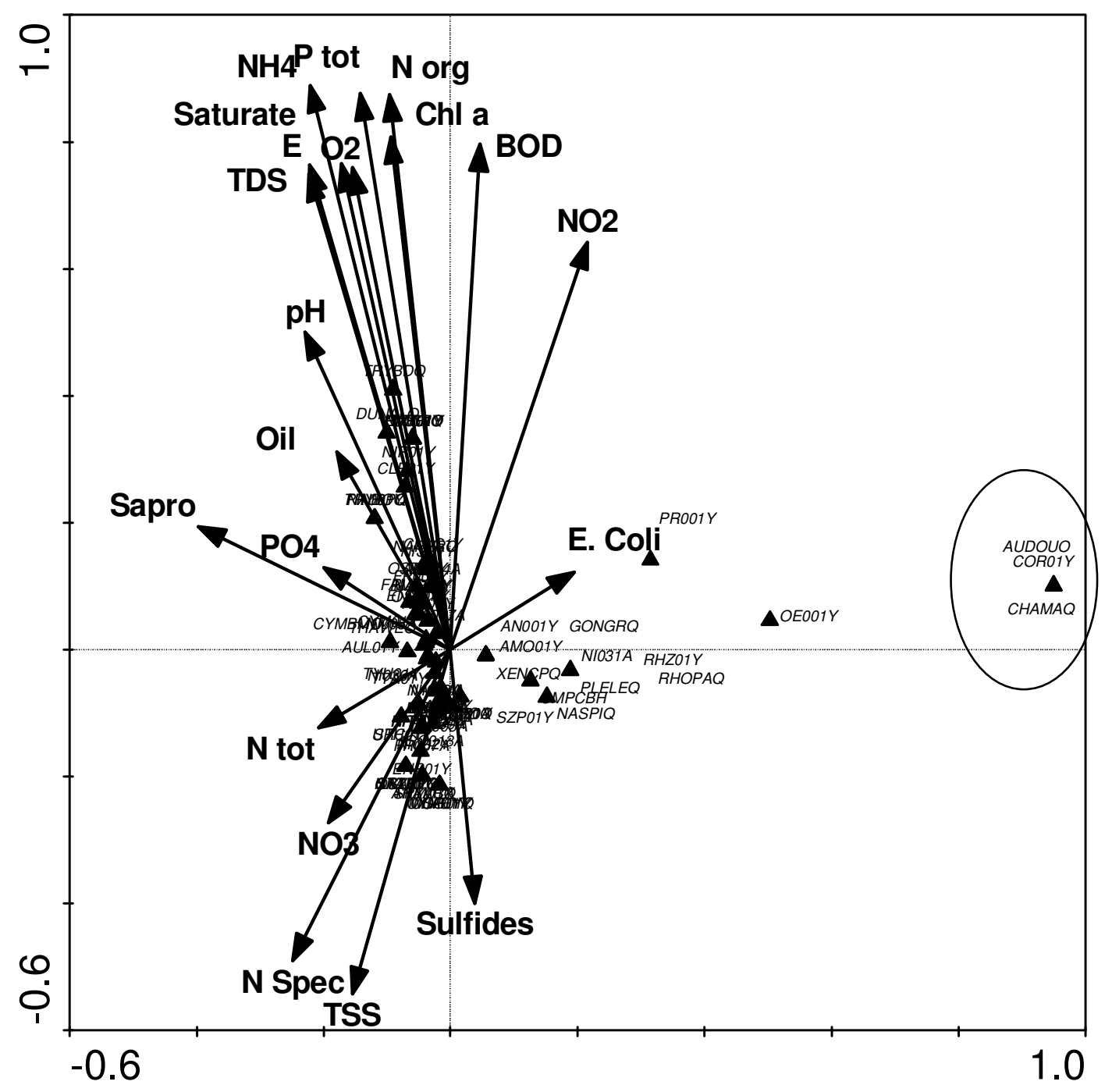

Figure 14. Biplot of environmental variables and species scores in a CCA of the Qishon River. Full names of species are presented in Table 3.

The pallet, Figure 14, gives the results of calculation of all environmental factors influencing the algal diversity in the Qishon River. According to the pallet the environmental factors can be divided into two separate groups. The first group includes factors of salinity and the pollution by ammonia, oil and sulfites, as well as the ecosystems activity factors - the concentrations of oxygen, nitrogen, phosphates, BOD and the chlorophyll $a$. The second group of factors includes nitrates and the related total nitrogen, as well as the water turbidity (TSS) and anoxia (sulfides) factors.

We identified a group of species that prefers clear and clean fresh water with low species diversity (right circle). This group includes the red algae Audouinella pygmaea, the seldom encountered green algae Characium ornithocephalum and the rare bluegreen species Chamaesiphon amethystinus. 


\section{Conclusion}

In two polluted rivers we revealed algal diversity consisting of 184 algal species and cyanoprokaryotes in the Rudnaya River, and 176 in the Qishon River. The diversity of species was higher in the Rudnaya River. Species diversity in the rivers is significantly different but the distribution of species over the higher taxa were very similar for both rivers.

Based upon the indicator analysis, accepted in the EC (European Parliament 2000), we conclude, that the water is cleaner and the algal diversity is higher in the Rudnaya River than in the Qishon River. The bioindication analysis of species preferences over the ecological groups in respect of salinity, acidification, and oxygenation shows similar distributions. Autecology of algal species is similar in both river communities but the Qishon is more alkalic and organically more polluted than the Rudnaya River. The indices of saprobity S affirm this conclusion. In addition, the Rudnaya River shows a weak 2-year cyclicity of organic pollution which may be a consequence of natural climatic cycles. The impact of pollution here increases downstream to the mouth, whereas the Qishon River is more polluted in the upper reaches, with periodically fluctuating pollution near the Haifa Chemicals. The impact of this pollution decreases down to the mouth.

The CCA determines the most significant factors affecting the species diversity in both rivers. As we know, the most effective application of this statistical approach is the comparison of communities' preferences from different phytogeographical provinces and seasons (Rédei et al. 2003).

As a result, we found a group of biosensors in Rudnaya River sensitive to borates and fluorides: Ankistrodesmus acicularis, A. angustus, Scenedesmus acuminatus, which are used in biotesting of toxicants, as well as Lyngbya kuetzingii, Neidium ampliatum, and Sellaphora rectangularis.Low clean water requiring species richness was identified in Qishon River. This group includes the red algae Audouinella pygmaea, low abounded green algae Characium ornithocephalum, as well as the rare blue-green species Chamaesiphon amethystinus. These species can be used as biosensors of high pollution in carbonate provinces. The similarity of species diversity distribution over the major bioindicating groups for the both rivers and the specificity of their response to the increasing pollutant concentrations leads us to the following conclusion.

We found that algal biodiversity is more sensitive to technogenic pollution in the silicate province being more tolerant to the same organic pollutants in the carbonate province.This can be related to the presence of metal ions in the water of silicate province with ph less than 7 . In contrast, in the carbonate province, the metals are precipitated and their toxic impact is lower.

Thus, our comparison of two polluted rivers shows, that the combination of bioindicational methods and statistics are effective to determine the main factors affecting algal diversity, and are helpful in recognition of indicators or biosensing species for the most important environmental variables.

Acknowledgements. We thank Moti Tavassi, who collaborated in collecting algal samples from the Qishon River. This work has been partially funded by the Israel Ministry of Absorption. 


\section{REFERENCES}

[1] Banerjee, M. \& Sharma, D. (2004): Regulation of phosphatase activity in Chroococcidiopsis isolates from two diverse habitats: effect of light, $\mathrm{pH}$ and temperature. - Applied Ecology and Environmental Research 2 (1): 71-82.

[2] Barinova, S.S. (1997): Morphology of connective spines in diatom algae of the genus Aulacoseira Thwaites. - Paleontological J. 31(2): 239-245.

[3] Barinova, S.S., Anissimova, O.V., Nevo, E., Jarygin, M.M. \& Wasser, S.P. (2004): Diversity and Ecology of Algae from Nahal Qishon, Northern Israel. - Plant Biosystems 138(3): 245-259.

[4] Barinova, S.S., Anissimova, O.V., Nevo, E., Wasser, S.P., Jarygin, M. (2006): Fresh and brackish water algae new for Israel found in Nahal Qishon (N-Israel). - Flora Mediterranea 15: 73-98.

[5] Barinova, S.S., Medvedeva, L.A. (1987): Biological analysis of water quality in major reservoirs of Primorsky region. - Bottom invertebrate of the rivers in Far East and East Siberia. The question of productivity and bioindication of pollution., Far-East Sci. Center of Russian Academy of Science. Vladivostok: 57-63 (Rus).

[6] Barinova, S.S., Medvedeva, L.A. (1989): Sanitary - biological analysis of modern state of the Rudnaya River. - Ecological Problems in Dalnegorsk Region and Possible Solutions (Abstr.). Sci. Appl. Seminar, 6-8 December 1989. Dalnegorsk: 22-23. (Rus).

[7] Barinova, S.S., Medvedeva, L.A. (1992a) A long-term assessment of seasonal pollution dynamics in the Rudnaya River, Primorskiy Region on the basis of algal diversity. Ecological Regulation. Problems and Methods. Inst. Nat. Cons. Moscow: 10-12. (Rus).

[8] Barinova, S.S., Medvedeva, L.A. (1992b): The comparative analysis of two methods of an estimation of water quality on algal saprobity. - The estimation of a condition and stability of ecosystems (Abstr.). Inst. Nat. Cons., Moscow: 41-43.

[9] Barinova, S.S., Medvedeva, L.A., Anissimova, O.V. (2006). Diversity of algal indicators in environmental assessment. - Pilies Studio, Tel Aviv. (In Russian); http://herba.msu.ru/algae/materials/book/title.html

[10] Berezneva T.Y. (2006): Rudnaya River. - www.Primpogoda.ru April 12, 2006.

[11] Elpatievsky, P.V., Chudaeva, V.A., Zazykina, E.F., Arzhanova, V.S. (1976): Role of the technogenic factor in formation of an ionic drain (on an example of one of the rivers of Primorski Region). - Geochemistry of a zone hypergenesis and technical activity of the peoples. Vladivostok: Far-East Scientific Center of Academy of Science of the USSR: 17-27.

[12] European Parliament (2000): Directive 2000/60/EC of the European Parliament and of the Council establishing a framework for Community action in the field of water policy, p. 1-72. - O.J. L 327.

[13] Herut B., Shefer E., Cohen Y. (2003): Environmental Quality of Israel's Mediterranean Coastal waters in 2002. Annual Report of the National Marine Environmental Monitoring Program. - IOLR Report H39/2003a.

[14] Herut, B., Hornung, H., Kress, N. (1994): Mercury, lead, copper, zinc and iron in shallow sediments of Haifa Bay, Israel. - Fresenius Environ. Bull. 3: 147-151.

[15] Herut, B., Kress, N. (1997): Particulate metals contamination in the Kishon River estuary, Israel. - Mar. Pollut. Bull. 34: 706-711.

[16] Herut, B., Kress, N., Hornung, H. (2000): Nutrient pollution at the lower reaches of Mediterranean coastal rivers in Israel. - Water Sci. Technol. 42: 147-152.

[17] Herut, B., Kress, N., Shefer, E., Hornung, H. (1999): Trace element levels in mollusks from clean and polluted coastal marine sites in the Mediterranean, Red and North Seas. Helgoland Mar. Res. 53: 154-162. 
[18] Kachur, A.N., Arzhanova, V.S., Yelpatyevsky, P.V., Von Braun, M.C. and Von Lindern I.H. (2003): Environmental conditions in the Rudnaya River watershed - a compilation of Soviet and post-Soviet era sampling around a lead smelter in the Russian Far East. - The Science of the Total Environment 303(1-2): 171-185.

[19] Kress, N., Herut, B., Shefer, E., Hornung, H. (1999): Trace element levels in fish from clean and polluted coastal marine sites in the Mediterranean Sea, Red Sea and North Sea. - Helgoland Mar. Res. 53: 163-170.

[20] Krumgalz, B. (1993): "Fingerprints" approach to the identification of anthropogenic trace metal sources in the nearshore and estuarine environments. - Estuaries 16: 488-495.

[21] Kuznetsova O. V. (2005): Industrial activity and the ecological problems in the Dalnegorsk region. - Proceedings Far-Eastern State Technical University. 131. Vladivostok.

[22] Medvedeva, L.A. Barinova, S.S., Kukharenko, L.A. (1986a): Algae of the Rudnaya River Basin (Primorskiy Region). - Flora and Systematics of Spore Plants in Far East. Vladivostok: Acad. Sci. USSR, Far East Branch: 36-48. (Rus).

[23] Medvedeva, L.A., Barinova, S.S., Kukharenko, L.A. (1986b): Sanitary and Biological characteristic of the Rudnaya River Basin. - Bentic Organisms in Fresh Waters of Far East. Vladivostok: Acad. Sci. USSR, Far East Branch: 108-115. (Rus).

[24] Medvedeva, L.A., Barinova, S.S. (1992): Seasonal changes of water quality in the Rudnaya River (Primorye Region). - The estimation of a condition and stability of ecosystems (Abstr.). Inst. Nat. Cons., Moscow: 43-45.

[25] Meybeck, M., Helmer, R. (1989): The quality of rivers: from pristine stage to global pollution. - Palaeogeography, Palaeoclimatology, Palaeoecology (Global and Planetary Change Section) 75: 283-309.

[26] Pantle, E., Buck, H. (1955): Die biologische Uberwachung der Gewässer und die Darstellung der Ergebnisse. - Gas- und Wasserfach 96(18): 1-604.

[27] Qishon River Authority. (2006): http://www.Qishon.org.il/.

[28] Rédei, D., Gaál, M., Hufnagel L. (2003): Spatial and temporal patterns of true bug assemblages extracted with Berlese funnels (data to the knowledge on ground-living Heteroptera of Hungary, № 2). - Applied Ecology and Environmental Research 1 (1-2): 115-142.

[29] Richter, E.D., Friedman, L.S., Tamir, Y., Berman, T., Levy, O., Westin, J.B., Peretz, T. (2003): Cancer risks in naval divers with multiple exposures to carcinogen. Environ. Health Perspect. 111: 609-617.

[30] Sharov, P.O. (2005): Lead Contamination of Environment in Rudnaya Pristan, Russia and associated Health Risks. Far Eastern Health Fund. - Vladivostok, Dalnauka.

[31] Shilov, V.N. (1997): Comparison of Neogene-Quaternary volcanism in Sakhalin and the East Sikhote Alin: 3. A comparative study of the rock chemistries. Journal of Volcanology and Seismology 18: 667-672.

[32] Sládeček, V. (1986): Diatom as indicators of organic pollution. - Arch. Hydrochim. Hydrobiol. 14: 555-566.

[33] Swift, E. (1967): Cleaning diatom frustules with ultraviolet radiation and peroxide. Phycologia 6: 161-163.

[34] ter Braak, C.J.F. (1987): The analysis of vegetation-environment relationships by canonical correspondence analysis. - Vegetatio 69: 69-77.

[35] ter Braak, C.J.F. (1990): Interpreting canonical correlation analysis through biplots of structural correlations and weights. - Psychometrica 55: 519-531.

[36] Von Braun, M.C., Von Lindern, I.H., Khristoforova, N.K. et al. (2002): Environmental lead contamination in the Rudnaya Pristan-Dalnegorsk mining and smelter district, Russian Far East. - Environmental Research, Section A 88: 164-173.

[37] Whitton, B.A., Roth, E.,Friedrich, G. (eds.). (1991): Use of algae for monitoring rivers, Institut für Botanik. - Univ. Press. Innsbruck. 